\title{
Modeling Financial System with Interbank Flows, Borrowing, and Investing
}

\author{
Aditya Maheshwari ${ }^{1}$ and Andrey Sarantsev ${ }^{2, *}$ \\ 1 Department of Statistics and Applied Probability, University of California, Santa Barbara, CA 93106, USA; \\ aditya_maheshwari@ucsb.edu \\ 2 Department of Mathematics and Statistics, University of Nevada, Reno, NV 89557, USA \\ * Correspondence: asarantsev@unr.edu
}

Received: 6 October 2018; Accepted: 13 November 2018; Published: 15 November 2018

check for updates

\begin{abstract}
In our model, private actors with interbank cash flows similar to, but more general than that by Carmona et al. (2013) borrow from the non-banking financial sector at a certain interest rate, controlled by the central bank, and invest in risky assets. Each private actor aims to maximize its expected terminal logarithmic wealth. The central bank, in turn, aims to control the overall economy by means of an exponential utility function. We solve all stochastic optimal control problems explicitly. We are able to recreate occasions such as liquidity trap. We study distribution of the number of defaults (net worth of a private actor going below a certain threshold).
\end{abstract}

Keywords: systemic risk; stochastic control; principal-agent problem; stochastic game; stationary distribution; stochastic stability; Lyapunov function

JEL Classification: C61; E43; E44; E52; G11

\section{Introduction}

We are interested in modeling interaction between utility-maximizing private actors (which for simplicity we call private banks or simply banks) and a central bank, which regulates borrowing activity via an interest rate. Private banks exchange (exogenous) cash flows, and borrow from the non-banking financial sector to invest in profitable but risky assets. This central bank can lower interest rate to stimulate financial activity by private actors, or increase this rate to cool this activity down. Sometimes, however, there are not many profitable investments. Then, the private actors do not borrow at all, while the central bank is not able to remedy this even by lowering the rate to zero; this is called the liquidity trap.

We mention the concept of systemic risk, which can be informally described as the probability of a large number of banks defaulting or getting into financial trouble. We understand default or failure of a bank as its net worth (assets minus liabilities) going below a given threshold. We are interested in probability of this undesirable event; of the mechanism of such failure; and of the financial contagion, when failure of a few banks leads to many more failures. We refer the reader to the handbook (Fouque and Langsam 2013) containing many different approaches to systemic risk. Our work is inspired by the model introduced in (Carmona et al. 2013) and also described in (Carmona 2016, sct. 5.5).

If $X_{i}(t)$ is the wealth and $Y_{i}(t):=\log X_{i}(t)$, authors in (Carmona et al. 2013) model the banking system as a system of $N$ continuous-time stochastic processes $Y_{1}, \ldots, Y_{N}$, with multidimensional Ornstein-Uhlenbeck dynamics. The stochastic differential equations are given by:

$$
\mathrm{d} Y_{i}(t)=a\left(\bar{Y}(t)-Y_{i}(t)\right) \mathrm{d} t+\sigma \mathrm{d} W_{i}(t), \quad i=1, \ldots, N,
$$


with i.i.d. (independent identically distributed) Brownian motions $W_{1}, \ldots, W_{N}$, constants $a, \sigma>0$, and

$$
\bar{Y}(t)=\frac{1}{N} \sum_{i=1}^{N} Y_{i}(t), \quad t \geq 0
$$

The constant $a$ is referred to as the interbankflow rate. In (Carmona et al. 2013), these mean-reverting drifts are generated by the decisions of banks to borrow money from one another. Their decisions are done by minimizing a certain cost functional, which measures, roughly speaking, the preference of a bank to borrow from other banks, as opposed to borrowing from the central bank. The authors discuss both the finite player solution and the mean-field limit of the problem in the context of systemic risk.

Remark 1. Apply Itô's formula to rewrite Equation (1) in terms of $X_{i}$ (the actual net worth of the ith bank) instead of $Y_{i}=\log X_{i}$. Then, the interbank flows derived from Ornstein-Uhlenbeck-type terms $a\left(\bar{Y}(t)-Y_{i}(t)\right) \mathrm{d} t$ do not add up to 0 . One can think that the remainder comes from (or to, depending on the sign) the real economy or non-banking financial sector. Nevertheless, the model in Equation (1) attracted a lot of attention because of its simplicity and analytical tractability.

We further explore the individual decision-making of the private banks and extend the role of the central bank. Furthermore, we analyze how this decision-making affects the stability of the system. More specifically, we extend the model by assuming that each private bank invests in a risky portfolio of assets, borrows money from the non-banking financial sector to invest in this portfolio (with interest rate controlled by the central bank), pockets the profit, and pays back the interest. Private banks want to maximize the terminal logarithmic wealth:

$$
\sup \mathbb{E}\left[\log X_{i}(T)\right]=\sup \mathbb{E}\left[Y_{i}(T)\right], i=1, \ldots, N,
$$

by borrowing and investing in a portfolio of risky assets. For simplicity, we assume the portfolios of private banks are correlated geometric Brownian motions. The choice of logarithmic utility function allows us to solve the corresponding Hamilton-Jacobi-Bellman (HJB) equation explicitly. Logarithmic utility, as shown in (Feldman 1992), corresponds to myopic decision-making; in other words, private actors are short-sighted.

Unlike (Carmona et al. 2013), where the decision making of the central bank is not analyzed, in our model the central bank uses interest rate $r \geq 0$ as a monetary policy instrument (to govern the behavior of the private banks). This is a solution of the control problem solved by the central bank:

$$
\mathbb{E}[-\exp (-\lambda \bar{Y}(T))] \text { for some } \lambda>0
$$

As we see later, the central bank is more risk-averse than private banks. Sometimes it needs to reduce overall risk by increasing the interest rate, which controls the overall size of the system, measured by $\bar{Y}$ from Equation (2). This is not the average net worth of banks; this is the average of the logarithms of net worth. This measure is somewhat non-standard; however, it is more appropriate for our model, when dynamics in Equation (1) is written in terms of logarithms $Y_{i}$ of net worth $X_{i}$. This measure is used in (Carmona et al. 2013) and subsequent papers, so we feel justified in using it here. Similar to the problem in Equation (3), we can solve Equation (4) explicitly, because of the choice of exponential utility. (Sometimes it is called CARA: constant absolute risk aversion utility function.) For other choices of utility functions, it is probably impossible to solve these optimal control problems explicitly. Then, one could possibly try to use mean-field limits, as in (Carmona et al. 2016; Carmona and Lacker 2015; Lacker 2016).

Remark 2. This setup resembles the principal-agent problem: the principal (now the central bank) allows private banks to borrow from the non-banking financial sector, and private banks (agents) maximize their expected logarithmic terminal utility (their contract). 
Under such optimal choices of the actors, we study the dynamics of logarithmic net worth of banks, and the distribution of defaults. A default of the $i$ th bank is understood in the same way as above: when $X_{i}(t)$, the net worth of this bank, falls below some fixed positive threshold. This leads us to understanding systemic risk in this model: how defaults of a few banks can lead to defaults of many other banks (see Section 3.4).

Besides incorporating the optimal strategy of the central bank, we also generalize the model in Equation (1) by allowing interbank flow rates from bank $i$ to bank $j$ to depend on the banks $i, j$, and on time $t$, denoting this rate by $c_{i j}(t)$. This heterogeneity, together with Ornstein-Uhlenbeck dynamics, resembles to some extent the model by (Kley et al. 2015). As we show in Section 4, the matrix $\left(c_{i j}(t)\right)$ of interbank flow rates corresponds to the stability of the system. We also allow for Brownian motions $W_{1}, \ldots, W_{N}$ to have drifts and to be correlated: that is, we assume $W=\left(W_{1}, \ldots, W_{N}\right)$ is an $N$-dimensional Brownian motion with drift vector $\mu$ and covariance matrix $A$.

\subsection{Existing Models and Contributions}

The literature on systemic risk is divided into two main categories: graph theoretic models and dynamic models using stochastic differential equations. Graph theoretic formulation of systemic risk came to prominence through Eisenberg and Noe (2001). Many works have extended their model and one of the latest works is Banerjee et al. (2018). Our work here is in the second group and inspired by Carmona et al. (2013). Some of the recent works in this direction are (Carmona et al. 2018; Sun 2018). Our work uniquely adds to the existing literature by viewing systemic risk as principal-agent problem with central bank as the principal and private banks as the agents. To our knowledge, we are the first to view the systemic risk through this lens. More specifically, our work adds to existing literature in the following ways:

- First, we extend the model in (Carmona et al. 2013) to include the central bank. This affects the response of the private banks who now make decisions considering the monetary policy of the central bank. Our model allows for the central bank to play a more active role in stabilizing the banking system. This important feature was missing in the work of (Carmona et al. 2013) where the central bank was considered merely as a clearing house.

- Second, we reformulate the problem discussed in (Carmona et al. 2013) as a principal-agent problem where both, the principal (central bank) and the agent (private banks) maximize their respective utility. We assume that the private banks are risk neutral and central bank as risk averse. This allows us to get closed form solutions for the optimal policy of both the players.

- Third, we generalize the flow rates in our model to be different for each pair of private banks. This generalization converts the representative agent model discussed in (Carmona et al. 2013) into a heterogeneous agent model. This heterogeneity demonstrates a unique feature of the banking system where banks with higher interbank flows are less likely to default than their counterparts with lower interbank flows.

- Finally, as we show in Section 2, the volatility of the wealth process of the private banks is controlled, in contrast to (Carmona et al. 2013) where the volatility is constant for each bank.

\subsection{Organization of the Paper}

In Section 2, we describe the model in terms of stochastic control problem for a system of stochastic differential equations. In Section 3, we solve the stochastic control problem for each private bank, and in Section 4, for the central bank (given optimal control for each private bank). In particular, in Section 3, we study distribution of the number of defaults. This is where we touch the concept of systemic risk: We are interested in its dependence on the parameters of the system, for example correlations between returns on various risky investments. Section 5 contains results on long-term stability of the system: the fact that the capitals of banks tend to stay close, as opposed to splitting into 
two or more groups. Section 6 is devoted to concluding remarks and suggestions for future research. The Appendix contains some technical proofs.

\subsection{Notation}

For a vector or a matrix $a$, its transpose is denoted by $a^{\prime}$. We usually think of vectors as column-vectors. The dot product of two vectors $a$ and $b$ is denoted by $a \cdot b$. The term standard Brownian motion stands for a one-dimensional Brownian motion with drift coefficient 0 and diffusion coefficient 1 . For $V \equiv 1$, this is called the total variation norm. Fix a dimension $N \geq 2$. Then, $e \in \mathbb{R}^{N}$ is a vector $(1, \ldots, 1)^{\prime}$ with unit components, and we define the following hyperplane in $\mathbb{R}^{N}$ :

$$
\Pi:=\left\{x \in \mathbb{R}^{N} \mid x \cdot e=0\right\}=\left\{x \in \mathbb{R}^{N} \mid x_{1}+\ldots+x_{N}=0\right\} .
$$

Define the (closed) ball of radius $r$ on $\Pi$ centered at the origin:

$$
\mathcal{B}(r):=\{x \in \Pi \mid\|x\| \leq r\} .
$$

The $(N-1)$-dimensional Lebesgue measure on $\Pi$ is denoted by $\operatorname{mes}_{\Pi}(\cdot)$. As mentioned above, the symbol $1(A)$ or $1_{A}$ stands for the indicator function of an event $A$.

\section{Description of the Model}

\subsection{Formal Description}

Consider a system of $N$ agents (we call them private banks) which continuously lend money to each other, borrow from the non-banking financial sector, pay back the interest, and invest in some risky portfolios.

We operate on filtered probability space $\left(\Omega, \mathcal{F},\left(\mathcal{F}_{t}\right)_{t \geq 0}, \mathbb{P}\right)$ with the filtration satisfying the usual conditions. All the processes which we consider are adapted to the $\left(\mathcal{F}_{t}\right)_{t \geq 0}$. Let $X_{i}(t)>0$ be the net worth (assets minus liabilities) of the $i$ th bank at time $t$, for $i=1, \ldots, N$. Let $Z_{i}(t)$ be the amount borrowed at the moment $t$ by the $i$ th private bank from the non-banking financial sector. Assume the interest rate for such borrowing is $r(t) \geq 0$, controlled by the central bank. Then, during the time interval $[t, t+\mathrm{d} t]$, the $i$ th bank pays back interest $r(t) Z_{i}(t) \mathrm{d} t$. At time $t$, the $i$ th bank has at its disposal the amount $X_{i}(t)+Z_{i}(t)$ : its own capital plus borrowed amount. This amount $Z_{i}(t) \geq 0$ is controlled by the $i$ th bank. Herein, we assume perfect competition in the interbank lending market. We also assume that there is no limit on the maximum borrowing of the private banks.

Alternatively, the $i$ th bank might decide to not borrow anything, and instead to even put aside some of its own money in cash (which does not earn any interest). This happens if the investment is not very profitable, or, more precisely, if the return does not outweigh the risk. In this case, we let $Z_{i}(t)<0$, and define $-Z_{i}(t)$ to be the quantity of cash put aside. The amount invested is still $X_{i}(t)+Z_{i}(t)$, but the bank does not pay or receive any interest.

We combine these two cases: the $i$ th bank invests the amount $X_{i}(t)+Z_{i}(t)$ at time $t$ into a risky portfolio, and pays interest $r(t)\left(Z_{i}(t)\right)_{+} \mathrm{d} t$ during the time interval $[t, t+\mathrm{d} t]$.

At time $t$, the $i$ th bank invests in a portfolio of risky assets with value $S_{i}(t)$. The $i$ th bank buys $\left(X_{i}(t)+Z_{i}(t)\right) / S_{i}(t)$ units of this portfolio. Net profit for the time interval $[t, t+\mathrm{d} t]$ is

$$
\left(X_{i}(t)+Z_{i}(t)\right) \frac{\mathrm{d} S_{i}(t)}{S_{i}(t)} .
$$

Combining all of the above, we get the following system of equations:

$$
\mathrm{d} X_{i}(t)=\left(X_{i}(t)+Z_{i}(t)\right) \frac{\mathrm{d} S_{i}(t)}{S_{i}(t)}-r(t)\left(Z_{i}(t)\right)_{+} \mathrm{d} t i=1, \ldots, N, \text { and } X_{i}(0)>0 .
$$


Next, we make some assumptions on $S_{i}$, the dynamics of the portfolio processes. A separate question is how banks construct these portfolios out of stocks and other risky assets. This question is separate from the topic of this paper, and we do not study it here. Instead, we assume that these are geometric Brownian motions. This assumption is very simplifying, but we believe it captures to some extent the features of portfolios. The processes

$$
M_{i}(t)=\int_{0}^{t} \frac{\mathrm{d} S_{i}(s)}{S_{i}(s)}, i=1, \ldots, N,
$$

form an $N$-dimensional Brownian motion with drift vector $\mu=\left(\mu_{1}, \ldots, \mu_{N}\right)$ and covariance matrix $A=\left(a_{i j}\right)_{i, j=1, \ldots, N}$. In particular, each $M_{i}, i=1, \ldots, N$, is a Brownian motion with drift coefficient $\mu_{i}$ and diffusion coefficient $\sigma_{i}^{2}:=a_{i i}$, so it can be represented as

$$
M_{i}(t)=\mu_{i} t+\sigma_{i} W_{i}(t),
$$

where $W_{i}$ is a one-dimensional standard Brownian motion. Although the portfolio process in Equation (7) is driven by only one Brownian motion, a more general representation:

$$
\frac{\mathrm{d} S_{i}(t)}{S_{i}(t)}=\mu_{i} d t+\sum_{j=1}^{m} \sigma_{i, j} \mathrm{~d} B_{j}(t)
$$

where $\left(B_{1}, \ldots, B_{m}\right)$ are Brownian motions, can also be considered in our framework. Since $\sigma_{i} \mathrm{~d} W_{i}(t):=\sum_{j=1}^{m} \sigma_{i, j} \mathrm{~d} B_{j}(t)$, but $W_{i}$ is also a Brownian motion, we fall back to the original portfolio process in Equation (7).

The covariance between Brownian motions $\left(W_{1}, \ldots, W_{N}\right)$ can be modeled in various ways. (1.a) All $W_{1}, \ldots, W_{N}$ are independent. Then, the matrix $A$ is diagonal:

$$
A=\operatorname{diag}\left(\sigma_{1}^{2}, \ldots, \sigma_{N}^{2}\right)
$$

This means that the portfolios of banks are independent.

(1.b) All $W_{1}, \ldots, W_{N}$ are the same: $W_{1}=W_{2}=\ldots=W_{N}$. This means that all banks, in fact, use the same portfolio, and they are perfectly correlated. Then, it makes sense to let $\mu_{1}=\ldots=\mu_{N}$ and $\sigma_{1}=\ldots=\sigma_{N}$.

(1.c) An intermediate case: for some i.i.d. Brownian motions $\tilde{W}_{i}, i=0, \ldots, N$, and some coefficients $\rho_{0}, \tilde{\rho}_{0}$ with $\rho_{0}^{2}+\tilde{\rho}_{0}^{2}=1$ we have:

$$
W_{i}(t):=\rho_{0} \tilde{W}_{i}(t)+\tilde{\rho}_{0} \tilde{W}_{0}(t), i=1, \ldots, N .
$$

One can also split $N$ banks into subsets and construct dependence as in Case (1.c) for each subset; portfolio processes corresponding to different subsets are assumed to be independent.

\subsection{Main System of Driving Stochastic Equations}

Apply Itô's formula to find the dynamics of $Y_{i}(t):=\log X_{i}(t)$ :

$$
\mathrm{d} Y_{i}(t)=\frac{\mathrm{d} X_{i}(t)}{X_{i}(t)}-\frac{\mathrm{d}\left\langle X_{i}\right\rangle_{t}}{2 X_{i}^{2}(t)}
$$

Combining Equation (6) with Equation (11), we get our main stochastic equation, driving banks' wealth. For now, it does not contain interbank flows, which are Ornstein-Uhlenbeck-type drifts as in Equation (1):

$$
\mathrm{d} Y_{i}(t)=\left(1+\alpha_{i}(t)\right) \sigma_{i} \mathrm{~d} W_{i}(t)+h_{i}\left(\alpha_{i}(t), r(t)\right) \mathrm{d} t .
$$


Here, we define the relative investment ratio:

$$
\alpha_{i}(t)=\frac{Z_{i}(t)}{X_{i}(t)}, t \geq 0, i=1, \ldots, N,
$$

and the following quantity:

$$
h_{i}(\alpha, r):=(1+\alpha) \mu_{i}-\frac{\sigma_{i}^{2}}{2}(1+\alpha)^{2}-r \alpha_{+} \text {for } \alpha, r \in \mathbb{R} .
$$

Finally, the ith bank also interacts with other banks, having cash flow in and out. In (Carmona et al. 2013) and subsequent papers, this interaction is modeled by Ornstein-Uhlenbecktype drifts

$$
a\left(\bar{Y}(t)-Y_{i}(t)\right)
$$

from Equation (1), with $Y_{i}(\cdot)=\log X_{i}(\cdot)$. Here, we take drifts

$$
\frac{1}{N} \sum_{j=1}^{N} c_{i j}(t)\left(Y_{j}(t)-Y_{i}(t)\right)
$$

which are more general than Equation (14), and add them to Equation (12). Here, we assume that the flow rates satisfy

$$
c_{i j}(t)=c_{j i}(t), i \neq j ; c_{i i}(t)=0, i=1, \ldots, N .
$$

Remark 3. While we generalize the model of (Carmona et al. 2013), the symmetric interbank flows is clearly a simplification of real banking network where the interbank flows tend to be asymmetric. However, this assumption simplifies the problem mathematically as the aggregate wealth $\bar{Y}(t)$ is not dependent on the interbank flows and its dynamics can be expressed through the stochastic differential Equation (35). Furthermore, this allows us to solve for the optimal control of the central bank in closed form.

Note that in our model, as in (Carmona et al. 2013), the cash flows (in the original scale, not logarithmic one) do not necessarily add up to zero. Consider possible particular cases:

(2.a) All $c_{i j}(t) \equiv 0$. Then, there are no cash flows between banks.

(2.b) All $c_{i j}(t) \equiv c(t)>0$. For a constant $c$, this is the model from (Carmona et al. 2013).

(2.c) Let $G$ be a graph on vertices $\{1, \ldots, N\}$. Fix a $c(t)>0$ for all $t>0$. Let

$$
c_{i j}(t)=\left\{\begin{array}{l}
c(t) \text { if the vertices } i, j, \text { are adjacent } \\
0 \text { else }
\end{array}\right.
$$

After superimposing these Ornstein-Uhlenbeck-type drifts from Equation (15) on top of Equation (12), our main driving equation takes the form

$$
\begin{array}{rl}
\mathrm{d} Y_{i}(t)=\left(1+\alpha_{i}(t)\right) \sigma_{i} & \mathrm{~d} W_{i}(t)+h_{i}\left(\alpha_{i}(t), r(t)\right) \mathrm{d} t \\
& +\frac{1}{N} \sum_{j=1}^{N} c_{i j}(t)\left(Y_{j}(t)-Y_{i}(t)\right) \mathrm{d} t, \quad i=1, \ldots, N .
\end{array}
$$

Equation (17) resembles the model from (Carmona et al. 2013). However, it also has significant differences: the volatility in Equation (17) can be controlled (bank $i$ controls its investment decisions via $\alpha_{i}(t)$ which drives the volatility of the process $Y_{i}(t)$ ), unlike in (Carmona et al. 2013); and the drift 
coefficient in Equation (17) is a bit more complicated. For homogeneous rates: $c_{i j}(t) \equiv c(t)$, Equation (17) takes the form

$$
\mathrm{d} Y_{i}(t)=\left(1+\alpha_{i}(t)\right) \sigma_{i} \mathrm{~d} W_{i}(t)+h_{i}\left(\alpha_{i}(t), r(t)\right) \mathrm{d} t+c(t)\left(\bar{Y}(t)-Y_{i}(t)\right) \mathrm{d} t
$$

for $i=1, \ldots, N$, where $\bar{Y}(t)$ is defined in Equation (2). Moreover, this modification makes the representative agent model as studied by (Carmona et al. 2013) into a heterogeneous agent model.

\subsection{Interpretation}

As in (Carmona et al. 2013), we consider bank $i$ to be in bankruptcy at time $t$ if $X_{i}(t)<e^{D}$, where $D$ is a given threshold, stipulated by the central bank. The central bank would like to stimulate the activity of banks by persuading them to take risks, but not too much, lest they may become bankrupt. Equation (12) means that the central bank can use interest rate $r(t)$ as a monetary policy tool to alter the behavior of the private-banks.

Assume that banks start borrowing too much money and investing them in risky assets (leveraging). By doing this, they increase their probability of default. Then, the central bank can raise this interest rate to discourage private banks from excessive borrowing. Conversely, if banks are too cautious in borrowing against future profits and risk-taking, then the central bank can stimulate them by lowering the interest rate. As we show below, this interest rate affects the overall state of the system.

The parameter $r(t)$ is determined by the central bank and given to all private banks. These private banks then determine the investment rates $\alpha_{i}(t)$, independently of each other. In light of decision-making of the banks, the central bank needs to determine optimal values of these parameters. This setup is similar to the principal-agent problem, but with many agents.

\section{Optimal Behavior of Private Banks}

\subsection{Statement of the Problem}

We assume that the $i$ th bank takes as given the capital of other banks: $X_{j}(t), j \neq i$ (or, equivalently, $Y_{j}(t):=\log X_{j}(t), j \neq i$ ), as well as the interest rate $r(t)$ (the instrument of the monetary policy). The bank is trying to choose the relative investment ratio $\alpha_{i}(t)$, or, equivalently, the amount borrowed $Z_{i}(t)$, to maximize its expected terminal logarithmic wealth:

$$
\sup _{\alpha_{i}} \mathbb{E}\left[\log X_{i}(T)\right],
$$

where the supremum in Equation (19) is taken over all bounded adapted controls $\alpha_{i}=\left(\alpha_{i}(t), 0 \leq t \leq\right.$ $T)$. Assume that the interest rate $r(t)$ is already set by the central bank. In this section, we solve this stochastic control problem explicitly. This corresponds to the agent's problem in the principal-agent framework. In the next section, we discuss the optimal policy choices of the central bank (the principal).

\subsection{Solution of the Problem}

This specific choice of the utility function, which is linear in $Y_{i}$, and the choice of the interbank flows, which are also linear in $Y_{i}$ in Equation (12), makes this optimization problem tractable.

Theorem 1. For the optimization problem stated in Equation (19), where $\alpha_{i}$ is bounded, adapted on $[0, T]$, the following value of $\alpha_{i}$ is optimal for the ith private bank:

$$
\alpha_{i}^{*}(t):=\left\{\begin{array}{l}
\left(\frac{\mu_{i}-r(t)}{\sigma_{i}^{2}}-1\right)_{+}, \quad \mu_{i} \geq \sigma_{i}^{2} \\
\frac{\mu_{i}}{\sigma_{i}^{2}}-1, \quad \mu_{i} \leq \sigma_{i}^{2} .
\end{array}\right.
$$


Remark 4. In particular, if $\mu_{i} \leq \sigma_{i}^{2}$, that is, the return on the investment does not outweigh its risks, then the ith bank does not borrow anything to invest. On the contrary, this bank sets aside money as cash. If $\mu_{i} \geq \sigma_{i}^{2}$, the investment is attractive for borrowing, but a high enough interest rate: $r(t) \geq \mu_{i}-\sigma_{i}^{2}$ can preclude the ith bank from borrowing; then this bank will invest only its own money into the portfolio. Only if the interest rate is low enough: $r(t)<\mu_{i}-\sigma_{i}^{2}$, the ith bank borrows money to invest.

Remark 5. Note that the optimal strategies Equation (20) do not depend on the flow rates $c_{i j}$, because of the special choice of logarithmic utility function, which is linear in $Y_{i}$. Although logarithmic utility function leads to myopic agents, this assumption is important for mathematical tractability of the results. Let us mention CRRA (Constant Relative Risk Aversion, which corresponds to power functions) utility, or DARA (Decreasing Absolute Risk Aversion) utility, suggested in the paper (Machina and Viscusi 2014). These are other utility functions used in the literature. However, we were unable to evaluate the optimal control for these even in the mean field case.

Proof. The dynamic programming principle tells us that the function

$$
\Phi_{i}(t, y):=\sup _{\alpha_{i}} \mathbb{E}\left[Y_{i}(T) \mid Y_{i}(t)=y\right]
$$

where we take the supremum over all $\alpha_{i}$ which are bounded and adapted on $[t, T]$, satisfies the Hamilton-Jacobi-Bellman (HJB) equation:

$$
\begin{aligned}
& \frac{\partial \Phi_{i}}{\partial t}(t, y)+\sup _{\alpha_{i} \in \mathbb{R}}\left[\frac{1}{2} \sum_{j=1}^{N} \sum_{k=1}^{N}\left(1+\alpha_{j}\right) a_{j k} \frac{\partial^{2} \Phi_{i}}{\partial y_{j} \partial y_{k}}(t, y)\right. \\
& \left.\quad+\sum_{j=1}^{N}\left[h_{j}\left(\alpha_{j}, r(t)\right)+\frac{1}{N} \sum_{k=1}^{N} c_{j k}(t)\left(y_{k}-y_{j}\right)\right] \frac{\partial \Phi_{i}}{\partial y_{j}}(t, y)\right]=0,
\end{aligned}
$$

with terminal condition $\Phi_{i}(T, y)=y_{i}$. We assume all $\alpha_{j}, j \neq i$ are already chosen. Try the following anzats, linear in $y_{j}$ :

$$
\Phi_{i}(t, y)=g_{i 0}(t)+\sum_{j=1}^{N} g_{i j}(t) y_{j}
$$

Because it is linear, the second-order derivatives in Equation (21) turn out to be zero. Therefore, the only term in Equation (21) which needs to be maximized is $h\left(\alpha_{i}, r(t)\right)$. The solution to this maximization problem is given by the value $\alpha_{i}^{*}$ from Equation (20). This is a simple algebraic exercise; detailed calculations are given in Lemma A3 in the Appendix. The maximal value of $h_{i}(\alpha, r(t))$ is

$$
h_{i}^{*}(t):=h_{i}\left(\alpha_{i}^{*}(t), r(t)\right)=\left\{\begin{array}{l}
r(t)+\frac{\left(\mu_{i}-r(t)\right)^{2}}{2 \sigma_{i}^{2}}, r(t) \leq \mu_{i}-\sigma_{i}^{2} \\
\mu_{i}-\frac{1}{2} \sigma_{i}^{2}, \quad r(t) \geq \mu_{i}-\sigma_{i}^{2} \geq 0 ; \\
\frac{\mu_{i}^{2}}{2 \sigma_{i}^{2}}, \quad \mu_{i} \leq \sigma_{i}^{2} .
\end{array}\right.
$$

This means that the $i$ th bank chooses the control value $\alpha_{i}:=\alpha_{i}^{*}$. This value is independent of terminal time $T$, and of the values of $Y_{j}, j=1, \ldots, N$. This corresponds to the classical solution of the Merton problem. If $r$ is constant (independent of $t$ ), then $\alpha_{i}^{*}$ and $h_{i}^{*}$ are also constant. Comparing Equation (22) with the terminal condition, we have:

$$
g_{i j}(T)=\delta_{i j}=\left\{\begin{array}{l}
1, i=j ; \\
0, i \neq j,
\end{array} \quad \text { for } j=0, \ldots, N\right.
$$


Next, plug the anzats in Equation (22) into Equation (21). Note that all second-order derivatives of the anzats in Equation (22) are equal to zero, and first-order derivatives are

$$
\frac{\partial \Phi_{i}}{\partial y_{j}}=g_{i j}(t), j=1, \ldots, N
$$

In addition, the time derivative of this value function $\Phi$ from Equation (22) is

$$
\frac{\partial \Phi}{\partial t}=g_{i 0}^{\prime}(t)+\sum_{j=1}^{N} g_{i j}^{\prime}(t) y_{j}
$$

Combining Equations (20), (23), (25) and (26), we get that the HJB Equation (21) takes the form

$$
g_{i 0}^{\prime}(t)+\sum_{j=1}^{N} g_{i j}^{\prime}(t) y_{j}+\sum_{j=1}^{N} h_{j}^{*}(t) g_{i j}(t)+\frac{1}{N} \sum_{j=1}^{N} \sum_{k=1}^{N} c_{j k}(t)\left(y_{k}-y_{j}\right) g_{i j}(t)=0 .
$$

Comparing coefficients in Equation (27) at each $y_{j}$, we see that

$$
g_{i j}^{\prime}(t)+\frac{1}{N} \sum_{k=1}^{N} g_{i k}(t) c_{j k}(t)-\frac{1}{N} \sum_{k=1}^{N} g_{i k}(t) c_{k j}(t)=0, j=1, \ldots, N .
$$

The free terms in Equation (27) sum up to

$$
g_{i 0}^{\prime}(t)+\sum_{j=1}^{N} h_{j}^{*}(t) g_{i j}(t)=0 .
$$

Together with terminal conditions in Equation (24), this Systems in Equations (28) and (29) of $N+1$ linear ODEs has a unique solution $g_{i 0}, \ldots, g_{i N}$. This solves the HJB equation.

To complete the proof, let us do the verification argument. Take a bounded adapted control $\alpha_{j}=\left(\alpha_{j}(t), 0 \leq t \leq T\right)$ for each $j=1, \ldots, N$. Apply Itô's formula for $\Phi_{i}(t, Y(t))$ :

$$
\begin{aligned}
\mathrm{d} \Phi_{i}(t, Y(t)) & =\left[\frac{\partial \Phi_{i}}{\partial t}(t, Y(t))+\frac{1}{2} \sum_{j=1}^{N} \sum_{k=1}^{N}\left(1+\alpha_{j}(t)\right) a_{j k} \frac{\partial^{2} \Phi_{i}}{\partial y_{j} \partial y_{k}}(t, Y(t))\right. \\
& \left.+\sum_{j=1}^{N}\left[h_{j}\left(\alpha_{j}(t), r(t)\right)+\frac{1}{N} \sum_{k=1}^{N} c_{j k}(t)\left(Y_{k}(t)-Y_{j}(t)\right)\right] \frac{\partial \Phi_{i}}{\partial y_{j}}(t, Y(t))\right] \mathrm{d} t \\
& +\sum_{j=1}^{N} \frac{\partial \Phi_{i}}{\partial y_{j}}(t, Y(t))\left(1+\alpha_{j}(t)\right) \mathrm{d} W_{j}(t) .
\end{aligned}
$$

Using the boundedness of $\alpha_{j}=\left(\alpha_{j}(t), 0 \leq t \leq T\right)$, we get that the stochastic integral term in Equation (30) has expectation zero. Combining Equation (21) with Equation, (30), we get that $\left(\Phi_{i}(t, Y(t)), t \geq 0\right)$ is a supermartingale for all admissible (adapted bounded) controls $\alpha_{i}$, but a martingale for the control $\alpha_{i}^{*}$. Recall that $\Phi_{i}(T, y)=y_{i}$. Therefore, $\mathbb{E} \Phi_{i}(0, Y(0)) \geq$ $\mathbb{E} \Phi_{i}(T, Y(T))=\mathbb{E} Y_{i}(T)$, with equality for the control $\alpha_{i}^{*}$. From here it immediately follows that $\alpha_{i}^{*}$ is indeed the optimal control. 


\subsection{The Dynamics of Banks under Their Optimal Investment Choices}

Under the optimal control in Equation (20), the processes $Y_{i}, i=1, \ldots, N$ (we denote them by $Y_{i}^{*}$ ) satisfy the following system of stochastic differential equations:

$$
\mathrm{d} Y_{i}^{*}(t)=\mathrm{d} M_{i}^{*}(t)+\frac{1}{N}\left[\sum_{j=1}^{N} c_{i j}(t)\left(Y_{j}^{*}(t)-Y_{i}^{*}(t)\right)\right] \mathrm{d} t, i=1, \ldots, N,
$$

where $M_{1}^{*}, \ldots, M_{N}^{*}$, are given by

$$
\mathrm{d} M_{i}^{*}(t)=h_{i}\left(\alpha_{i}^{*}(t), r(t)\right) \mathrm{d} t+\sigma_{i}\left(1+\alpha_{i}^{*}(t)\right) \mathrm{d} W_{i}(t) .
$$

If $r=$ const, then $M^{*}$ is an $N$-dimensional Brownian motion with drift vector and covariance matrix given by

$$
\begin{gathered}
\mu^{*}=\left(\mu_{1}^{*}, \ldots, \mu_{N}^{*}\right), \mu_{i}^{*}:=h_{i}\left(\alpha_{i}^{*}, r\right) . \\
A^{*}:=\left(a_{i j}^{*}\right)_{i, j=1, \ldots, N}=\operatorname{diag}\left(\left(1+\alpha_{i}^{*}\right)^{2}, i=1, \ldots, N\right) A .
\end{gathered}
$$

The dynamics in Equation (31) is similar to that in (Carmona et al. 2013). If $r(t)$ does not depend on $t$, then $M^{*}=\left(M_{1}^{*}, \ldots, M_{N}^{*}\right)^{\prime}$, similar to $\left(M_{1}, \ldots, M_{N}\right)^{\prime}$, is an $N$-dimensional Brownian motion, but with different drift vector and covariance matrix. As in Equation (2), we define

$$
\bar{Y}^{*}(t)=\frac{1}{N} \sum_{i=1}^{N} Y_{i}^{*}(t)
$$

Averaging Equation (31) and using the symmetry property $c_{i j}=c_{j i}$, we have:

$$
\bar{Y}^{*}(t)=\frac{1}{N} \sum_{i=1}^{N} M_{i}^{*}(t)
$$

The interest rate $r$ controls the overall size of the system, measured by $\bar{Y}$. Express Equation (34) as:

$$
\mathrm{d} \bar{Y}^{*}(t)=g(r(t)) \mathrm{d} t+\rho(r(t)) \mathrm{d} \bar{W}(t),
$$

where $\bar{W}$ is a standard Brownian motion, and the coefficients $g(\cdot)$ and $\rho(\cdot)$ are defined as:

$$
\begin{gathered}
g(r):=\frac{1}{N} \sum_{i=1}^{N} g_{i}(r), g_{i}(r):=\left\{\begin{array}{l}
\frac{\left(\mu_{i}-r\right)^{2}}{2 \sigma_{i}^{2}}+r, r \leq \mu_{i}-\sigma_{i}^{2} ; \\
\mu_{i}-\frac{\sigma_{i}^{2}}{2}, r \geq \mu_{i}-\sigma_{i}^{2} ; \\
\frac{\mu_{i}^{2}}{2 \sigma_{i}^{2}}, \mu_{i}<\sigma_{i}^{2} .
\end{array}\right. \\
\rho^{2}(r):=\frac{1}{N^{2}} \sum_{i=1}^{N} \sum_{j=1}^{N} a_{i j} \rho_{i}(r) \rho_{j}(r), \rho_{i}(r):=\left\{\begin{array}{l}
\frac{\mu_{i}-r}{\sigma_{i}^{2}}, 0 \leq r \leq \mu_{i}-\sigma_{i}^{2} ; \\
1,0 \leq \mu_{i}-\sigma_{i}^{2} \leq r ; \\
\frac{\mu_{i}}{\sigma_{i}^{2}}, \mu_{i} \leq \sigma_{i}^{2} .
\end{array}\right.
\end{gathered}
$$

To illustrate the optimal choice of the investment ratio $\alpha_{i}^{*}=\alpha_{i}, i=1, \ldots, N$, we made some simulations. Take $N=30$ banks, with $\mu_{i}, \sigma_{i}, i=1, \ldots, N$ i.i.d. uniform $[0.1,0.2]$. Then, $\mu_{i} \geq \sigma_{i}^{2}$ for all $i$; that is, all portfolios are profitable to invest, at least for zero interest rate $r=0$. First, in Figure 1, we assume Equation (9), that is, the portfolio processes $S_{1}, \ldots, S_{N}$, are independent. We also assume that there are no flows:

$$
c_{i j}(t) \equiv 0, i, j=1, \ldots, N \text {. }
$$


We take three interest rates $r: 0 \%, 12 \%$, and 20\%, respectively. As expected, increasing the interest rate forces the banks to borrow less and thus optimal investment ratio $\alpha^{*}$ becomes 0 in Figure 1c while it varied between 2 to 12 in Figure 1a.

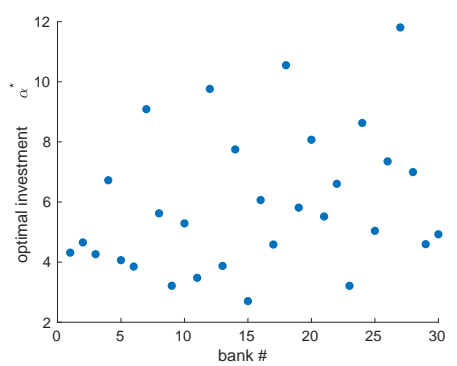

(a) $r=0$

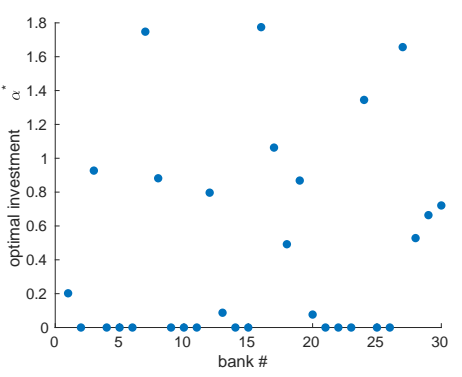

(b) $r=0.12$

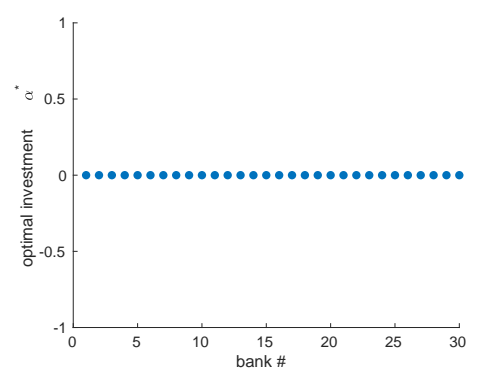

(c) $r=0.20$

Figure 1. We use the following parameters for the simulations: $N=30$ bank, time horizon $T=1$, no correlation $\rho_{0}=0$, no interbank flows $c_{i, j}=0,1000$ time steps and $\mu_{i}, \sigma_{i}, i=1, \ldots, N$ i.i.d. uniform $[0.1,0.2]$.

Next, in Figure 2, we assume portfolio processes are independent, as in Equation (9), but there are flows:

$$
c_{i j}=\left\{\begin{array}{l}
10, i, j=1, \ldots, 10 ; \\
0.5, \text { else. }
\end{array}\right.
$$

We observe that the banks with significant flows tend to have wealth dynamics which are more "tied" together. Moreover, this adds to the stability to the system as we observe lesser defaults for $i=1, \ldots, 10$ compared to $j=11, \ldots, 30$.

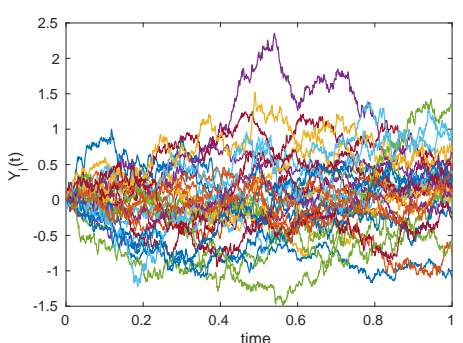

(a) All the banks

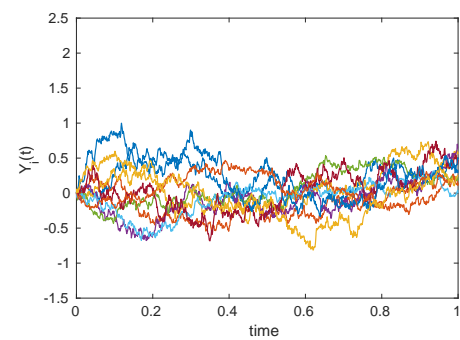

(b) $i=1, \ldots, 10$

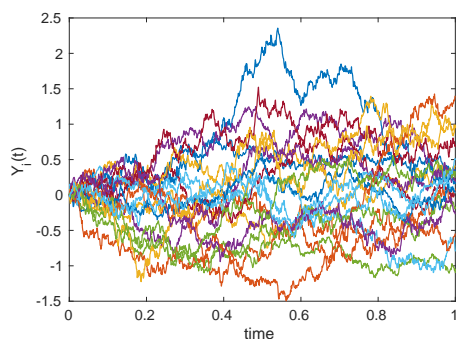

(c) $j=11, \ldots, 30$

Figure 2. Evolution of the logarithmic capital $Y_{i}(t)$ of banks, $i=1, \ldots, N$. We use the following parameters: interest rate $r=0, N=30$ banks, time horizon $T=1$, no correlation: $\rho_{0}=0$, interbank flows $c_{i, j}$ are as in Equation (38), 1000 time steps, and $\mu_{i}, \sigma_{i}, i=1, \ldots, N$ are i.i.d. uniform on $[0.1,0.2]$.

Finally, in Figure 3, we assume that the portfolio processes are correlated, as in Equation (10), with $\rho_{0}:=0.5$, flows are given by Equation (38) and interest rate $r$ is $8 \%$. Compared to Figure 2, the impact of correlation on the dynamics of the banks is clearly visible through movement of the wealth dynamics strongly tied together. 


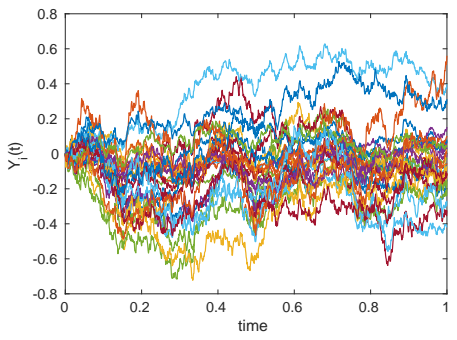

(a) All the banks

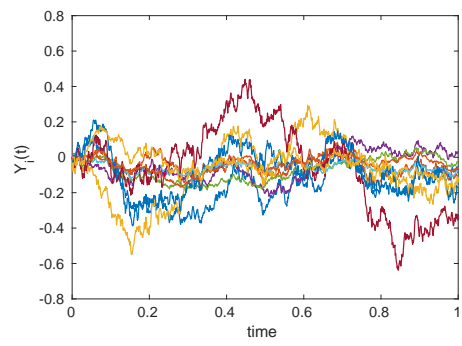

(b) $i=1, \ldots, 10$

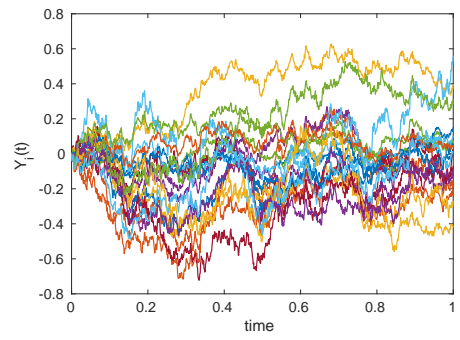

(c) $i=11, \ldots, 30$

Figure 3. Evolution of $Y_{i}(t)$, which represents the log capital of the $i$ th bank. We use the following parameters: interest rate $r=8 \%, N=30$ banks, time horizon $T=1$, correlation coefficient $\rho_{0}=0.5$, interbank flows $c_{i, j}$ as in Equation (38), 1000 time steps and $\mu_{i}, \sigma_{i}, i=1, \ldots, N$ i.i.d. uniform $[0.1,0.2]$.

\subsection{Systemic Risk}

Much of current research is devoted to systemic risk, that is, the probability of multiple bank defaults, and propagation of defaults through the system (in other words, contagion). To illustrate the probability of default of banks under different scenarios, we present the histogram and the empirical cumulative distribution function for number of defaults with $N=100$ banks and 1000 simulations. We assume the default threshold $D=-1$ in logarithmic wealth. That is, firms default if $Y_{i}(t)<D$ for some $t \in[0, T]$. Denote the (random) number $\mathcal{D}$ of defaults:

$$
\mathcal{D}:=\sum_{i=1}^{N} 1\left(\min _{0 \leq t \leq T} Y_{i}(t)<D\right)
$$

First, in Figure 4, we assume no interbank flows and independent portfolio process under different interest rate scenarios.

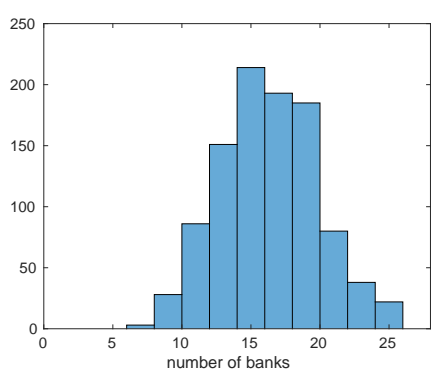

(a) $r=0$

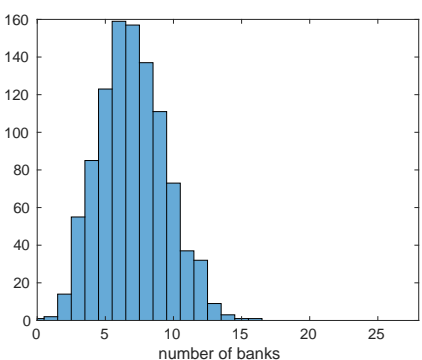

(b) $r=0.05$

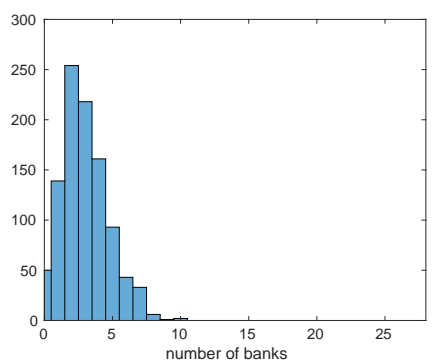

(c) $r=0.08$

Figure 4. Number of banks in default, whose log capital $Y_{i}(t)$ at some time $t \in[0, T]$ goes below $D=-1$. We use the following parameters: $N=100$ banks, 1000 simulations, no correlation: $a_{i j}=\sigma_{i}^{2} \delta_{i j}$, no interbank flows: $c_{i j}=0$ for $i, j=1, \ldots, N, 100$ time steps, and $\mu_{i}, \sigma_{i}, i=1, \ldots, N$ are i.i.d. uniform on $[0.1,0.2]$.

Next, in Figure 5, we present empirical cumulative distribution function (CDF) for the number of defaulted banks assuming correlated portfolios and no interbank flows for different interest rates. The corresponding histogram is presented in Figure 6. As stated in previous studies, increase in correlation increases the probability of large defaults and at the same time reducing the small default probabilities, similar to flocking behavior in various biological studies. Thus, in Figure 7, we present empirical estimates of $\mathbf{P}(\mathcal{D}>60)$ and $\mathbf{P}(\mathcal{D}<5)$, for $N=100$ banks, as a function of the correlation between their portfolio process at different interest rates. As expected, we observe the increase in probability of large and small default as the correlation increases. However, increasing the interest rate reduces the probability of large default at the expense of small default probability. Finally, in Figure 8, 
we present the empirical CDF for the number of defaulted banks assuming correlated portfolio process and constant interbank flows $c_{i j}=a$ for $i, j=1, \ldots, N$, where $a \in\{0,0.5,1\}$. We observe that interbank flows help stabilize the system and reduce the probability of default.

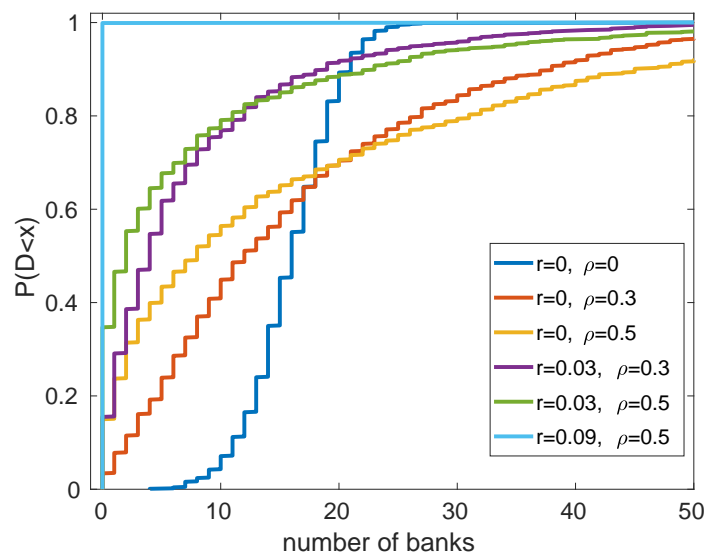

Figure 5. Empirical CDF of $\mathfrak{D}$, the number of banks in default, with $N=100$ banks, 1000 simulations, $\mu_{i}=\sigma_{i}=0.1$ for $i=1, \ldots, N$.

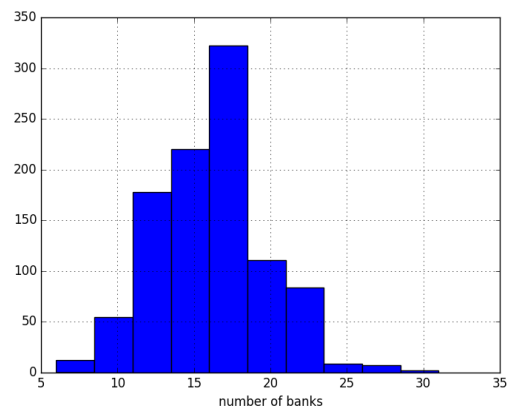

(a) $r=0$ and $\rho_{0}=0$

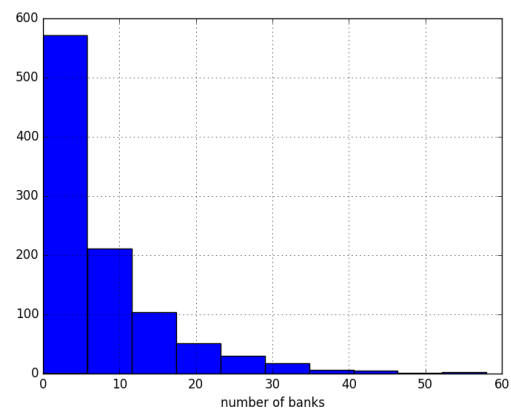

(c) $r=0.03$ and $\rho_{0}=0.3$

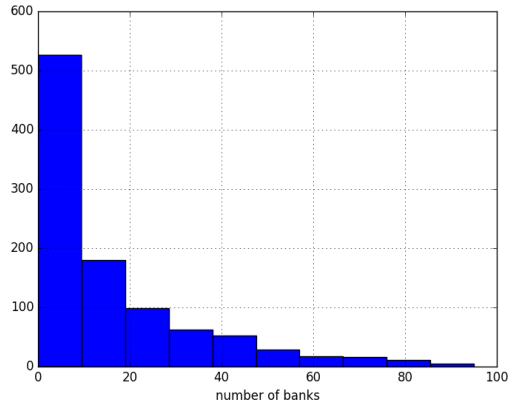

(b) $r=0$ and $\rho_{0}=0.5$

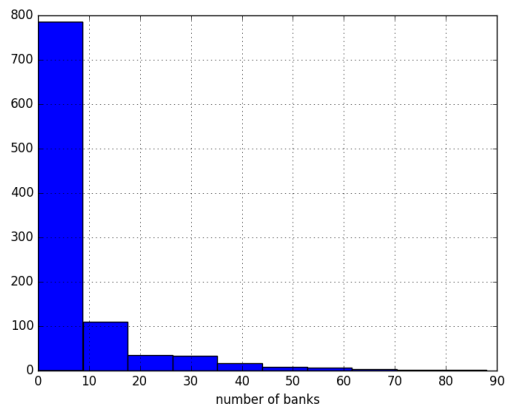

(d) $r=0.05$ and $\rho_{0}=0.3$

Figure 6. Histogram of the number of banks defaulting. We use the following parameters: $N=100$ banks, 1000 simulations, $\mu_{i}=\sigma_{i}=0.1$ for $i=1, \ldots, N$, and no interbank flows: $c_{i j}=0$ for $i, j=1, \ldots, N$. 


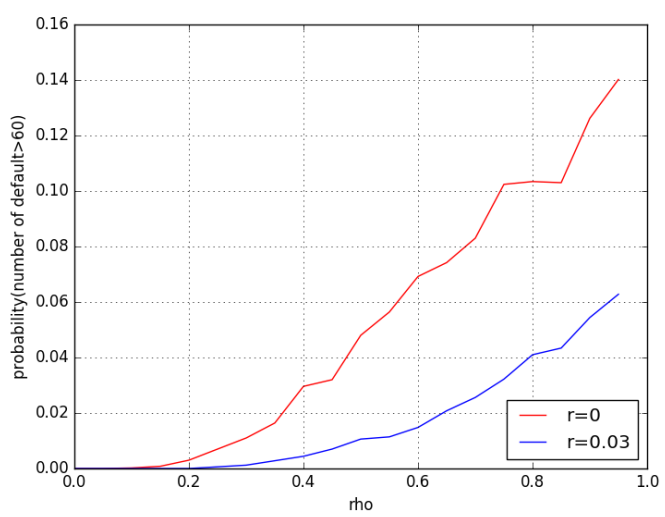

(a) Probability of a large default: $\mathcal{D}>60$

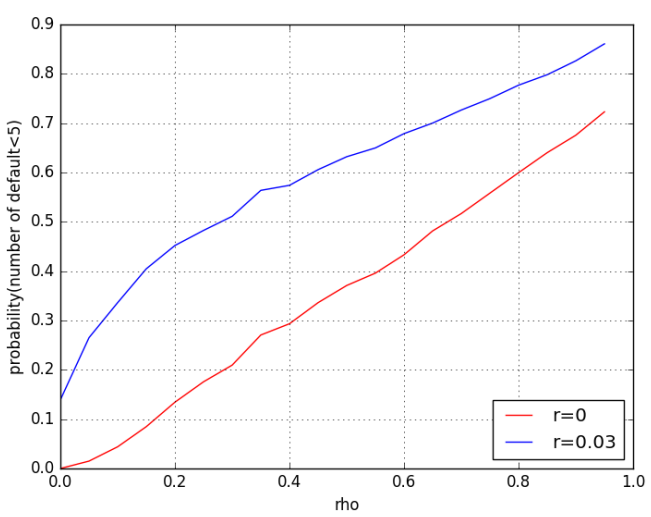

(b) Probability of a small default: $\mathcal{D}<5$

Figure 7. Empirical estimates of the probabilities of large and small defaults: $\mathbf{P}(\mathcal{D}>60)$ and $\mathbf{P}(\mathcal{D}<5)$, respectively, as a function of correlation between portfolio process $\rho_{0}$ at different interest rates. We use the following parameters: $N=100$ banks, 5000 simulations, $\mu_{i}=\sigma_{i}=0.1$ for $i=1, \ldots, N$, and interbank flow rates $c_{i j}=0$ for $i, j=1, \ldots, N$.

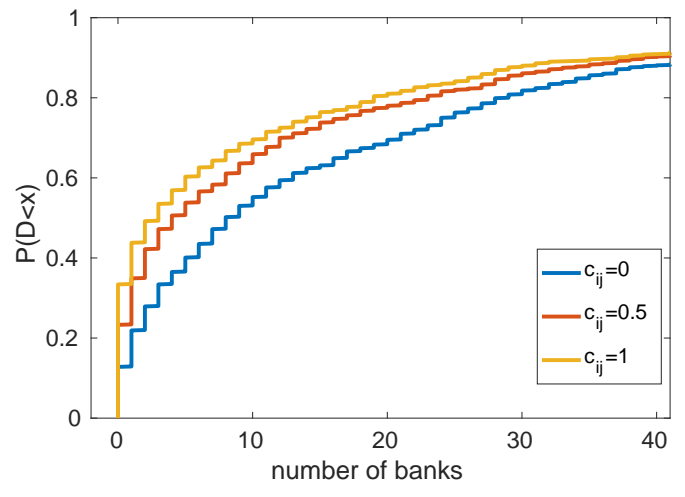

(a) $\rho_{0}=0.5$ and $r=0$

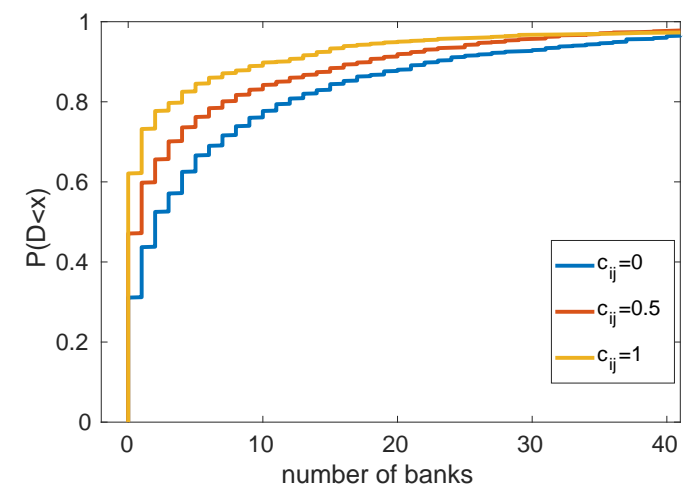

(b) $\rho_{0}=0.5$ and $r=0.03$

Figure 8. Empirical CDF of the number $\mathcal{D}$ of banks in default. We use the following parameters: $N=100$ banks, 1000 simulations, $\mu_{i}=\sigma_{i}=0.1$ for $i=1, \ldots, N$, and constant interbank flows $c_{i j}=a$ for $i, j=1, \ldots, N$, where $a \in\{0,0.5,1\}$.

\section{Optimal Central Bank Policy}

In this section, we assume that the central bank has to choose the interest rate $r$ in an optimal way, so that, after banks make their choice as in the previous section, optimal policy choice is achieved. We assume that banks make optimal (for them) choices and we omit all asterisks from notation of processes. This can be thought of as a principal's problem within the principal-agent problem framework. Let us now revisit the description of policy making by the central bank.

Its tool is the interest rate $r$, which the central bank uses to control the overall amount of capital in the system, measured by the $\bar{Y}^{*}$ from Equation (35). If the interest rate is low, the growth rate $g(r)$ from Equation (36) and the volatility $\rho^{2}(r)$ from Equation (37) are large. A more risk-averse central bank can choose therefore a larger $r$. One can apply a concave utility function to $\bar{Y}^{*}(t)$, and solve the stochastic control problem for this $r$. We apply the exponential (CARA: constant relative risk aversion) utility function to $\bar{Y}^{*}(t)$. 
The private banks wish to maximize their expected logarithmic net worth $Y_{i}(t)$. However, in terms of logarithmic capital, their utility function is linear, thus the private banks are risk neutral. Now, if the central bank was risk neutral, similar to the private banks, she would also try to maximize

$$
\mathbb{E}\left(Y_{1}(T)+\ldots+Y_{N}(T)\right) \text {, or, alternatively, } \mathbb{E} \bar{Y}(T),
$$

for a time horizon $T>0$. Below, we show that the central bank would then choose zero interest rate $r=0$, because this would produce the same result as the private banks were aiming for.

Now, suppose the central bank is risk-averse. This should manifest itself in the utility function being concave (rather than linear). Consider, for example, a commonly used exponential (CARA) utility function:

$$
U_{\lambda}(y):=-e^{-\lambda y} .
$$

Assume the central bank maximizes expected terminal utility:

$$
\sup _{r} \mathbb{E} U_{\lambda}(\bar{Y}(T)),
$$

where the supremum in Equation (41) is chosen over all bounded adapted controls $r$. We can alternatively choose instead of Equation (40) the utility function as

$$
U_{\lambda}(y)=\frac{1}{\lambda}\left(1-e^{-\lambda y}\right) .
$$

There is no difference between Equation (40) and Equation (42) when we try to maximize Equation (41), but writing Equation (42) highlights the risk-aversion of the central bank. As $\lambda \downarrow 0$, the function $U_{\lambda}$ from Equation (42) satisfies:

$$
U_{\lambda}(y) \rightarrow y
$$

The commonly used absolute risk aversion is calculated for Equation (42) as follows:

$$
-\frac{U_{\lambda}^{\prime \prime}(y)}{U_{\lambda}^{\prime}(y)}=\lambda
$$

In other words, $\lambda>0$ is the coefficient of risk aversion (of the central bank relative to private banks). For $\lambda=0$, the central bank is risk neutral.

Theorem 2. An optimal interest rate $r(t)$ for the problem in Equation (41) is given by a constant $r=r^{*}$ which maximizes the following expression:

$$
w(r, \lambda):=g(r)-\frac{\lambda}{2} \rho^{2}(r) .
$$

Remark 6. It is interesting to note that the optimal interest rate $r$ does not depend on the flow rates $c_{i j}$. This is because we measure the size of the system by the stochastic process $\bar{Y}(t)$. This process satisfies a stochastic differential equation with coefficients independent of $c_{i j}$. These coefficients do depend on the optimal controls $\alpha_{i}^{*}$. However, as we mentioned in Remark 5, these optimal controls $\alpha_{i}^{*}$, in turn, do not depend on the flow rates, because of our special choice of logarithmic utility function. We want to emphasize that in practice we expect to observe the interbank flow rate to affect the optimal interest rate. Particularly, asymmetric interbank flow rates are more likely to be one of the control variables for the corresponding bank, driving its wealth and consequently affecting the optimal interest rate. The result here is driven by the assumption of symmetric flows i.e., $c_{i j}=c_{j i}$. 
Proof. The HJB equation for the function

$$
\Phi(t, y):=\sup _{r} \mathbb{E}\left[U_{\lambda}(\bar{Y}(T)) \mid \bar{Y}(t)=y\right]
$$

where the supremum is taken over all bounded adapted controls $r=(r(t), 0 \leq t \leq T)$, takes the form

$$
\frac{\partial \Phi}{\partial t}+\sup _{r \geq 0}\left[\frac{\partial \Phi}{\partial y} g(r)+\frac{1}{2} \frac{\partial^{2} \Phi}{\partial y^{2}} \rho^{2}(r)\right]=0,
$$

with terminal condition $\Phi(T, y)=U_{\lambda}(y)$. Try the following form:

$$
\Phi(t, y)=f(t) U_{\lambda}(y) .
$$

From Equation (45), we can calculate derivatives with respect to $t$ and $y$ :

$$
\frac{\partial \Phi}{\partial t}=f^{\prime}(t) U_{\lambda}(y), \frac{\partial \Phi}{\partial y}=-\lambda \Phi, \frac{\partial^{2} \Phi}{\partial y^{2}}=\lambda^{2} \Phi .
$$

Plug Equation (46) into Equation (44). Because $\Phi<0$, we can rewrite Equation (44) as

$$
f^{\prime}(t)+f(t) \cdot \inf _{r \geq 0}\left[-\lambda g(r)+\frac{\lambda^{2}}{2} \rho^{2}(r)\right]=0 .
$$

This, in turn, is equivalent to

$$
\frac{f^{\prime}(t)}{\lambda f(t)}=\sup _{r \geq 0}\left[g(r)-\frac{\lambda}{2} \rho^{2}(r)\right]=: k_{0} .
$$

Since we have $\Phi(T, y)<0$ and $U_{\lambda}(y)<0$, for compatibility we need to show that $f(t)>0$ for all $t$. From the terminal condition $\Phi(T, y)=U_{\lambda}(y)$ combined with Equation (45), we have: $f(T)=1$. Equation (47) can be written as $f^{\prime}(t)=\lambda k_{0} f(t)$, which gives us $f(t)=\exp \left(\lambda k_{0}(t-T)\right)$. Therefore, $f(t)$ is positive.

Finally, let us do the verification argument to complete the proof. The idea is similar to the verification argument in Theorem 1 . Assume $r_{*}=\left(r_{*}(t), 0 \leq t \leq T\right)$ is our constant control from Equation (43), found from Equation (44), and $r=(r(t), 0 \leq t \leq T)$ is some other admissible (adapted bounded) control. Apply the function $\Phi(t, \cdot)$ to the process $\bar{Y}$. By Itô's formula,

$$
\begin{aligned}
\mathrm{d} \Phi(t, \bar{Y}(t))=\left[\frac{\partial \Phi}{\partial t}(t, \bar{Y}(t))\right. & \left.+\frac{\partial \Phi}{\partial y}(t, \bar{Y}(t)) g(r(t))+\frac{1}{2} \frac{\partial^{2} \Phi}{\partial y^{2}}(t, \bar{Y}(t)) \rho^{2}(r(t))\right] \mathrm{d} t \\
& +\frac{\partial \Phi}{\partial y}(t, \bar{Y}(t)) \rho(r(t)) \mathrm{d} \bar{W}(t) .
\end{aligned}
$$

Comparing Equation (44) with Equation (48), we get that $\Phi(t, \bar{Y}(t))$ is a supermartingale for the control $r$, but a martingale for the control $r_{*}$. Indeed, by boundedness of $r(t)$, the expectation of the stochastic integral in Equation (48) is zero. Since $\Phi(T, y)=U_{\lambda}(y)$, we get: $\mathbb{E} U_{\lambda}(\bar{Y}(T))=$ $\mathbb{E} \Phi(T, \bar{Y}(T)) \leq \mathbb{E} \Phi(0, \bar{Y}(0))$, with equality for the control $r_{*}$. The result immediately follows from here.

Let us find the $r$ which corresponds to the maximum in the right-hand side of (47). This depends on the structure of the vector $g$ and the matrix $A$.

If $\mu_{i} \leq \sigma_{i}^{2}$ for all $i=1, \ldots, N$, then all investments are too unprofitable to borrow money for them. Then, the interest rate policy cannot influence the behavior of private banks. This corresponds to the 
case of the liquidity trap, when conventional monetary policy no longer works. From now on until the end of this section, let us assume that all investments are attractive:

$$
\mu_{i} \geq \sigma_{i}^{2}, i=1, \ldots, N
$$

(3.a) Assume $S_{1}=\ldots=S_{N}$ : all investments are the same. Then, we have:

$$
\begin{gathered}
g_{1}=\ldots=g_{n}=: g, \text { and } \sigma_{1}=\ldots=\sigma_{N}=: \sigma ; \\
g(r)-\frac{\lambda}{2} \rho^{2}(r)=\left\{\begin{array}{l}
\frac{(\mu-r)^{2}}{2 \sigma^{2}}(1-\lambda), r \leq \mu-\sigma^{2} ; \\
\mu-\frac{\sigma^{2}}{2}(1+\lambda), r \geq \mu-\sigma^{2} .
\end{array}\right.
\end{gathered}
$$

The maximum is attained at $r=0$ for

$$
\lambda<\lambda_{*}:=1-2\left(\frac{\mu}{\sigma^{2}}+1\right)^{-1},
$$

and at any $r \geq \mu-\sigma^{2}$ for $\lambda>\lambda_{*}$. This has the following meaning: the case $\lambda<\lambda_{*}$ corresponds to less risk-averse central bank, and, to increase the total quantity of capital in the system, it wishes to slash the interest rate to zero. For the case $\lambda>\lambda_{*}$, however, the central bank is very risk-averse, and it increases the interest rate to prevent excessive borrowing and overheating of the financial system.

(3.b) Independent portfolio process: $a_{i j}=\sigma_{i}^{2} \delta_{i j}$, where $\delta_{i j}$ is as defined in Equation (24). Then,

$$
g(r)-\frac{\lambda}{2} \rho^{2}(r)=\frac{1}{N} \sum_{i=1}^{N}\left[g_{i}(r)-\frac{\lambda}{2 N} \sigma_{i}^{2} \rho_{i}^{2}(r)\right] .
$$

This function attains maximum:

$$
\begin{gathered}
\text { at } r=0 \text { for } \lambda<\lambda_{\min }:=N \min _{i=1, \ldots, N}\left[1-2\left(\frac{\mu_{i}}{\sigma_{i}^{2}}+1\right)^{-1}\right], \\
\text { at } r=\max _{i=1, \ldots, N}\left[\mu_{i}-\sigma_{i}^{2}\right] \text { for } \lambda>\lambda_{\max }:=N \max _{i=1, \ldots, N}\left[1-2\left(\frac{\mu_{i}}{\sigma_{i}^{2}}+1\right)^{-1}\right] .
\end{gathered}
$$

In the general case, we do not have an explicit form for the optimal $r$ in case $\lambda \in\left[\lambda_{\min }, \lambda_{\max }\right]$. If $\mu_{1}=\ldots=\mu_{N}=\mu$ and $\sigma_{1}=\ldots=\sigma_{N}=\sigma$, we have $\lambda_{\min }=\lambda_{\max }$. Note that here the central bank chooses expansionary monetary policy (zero interest rate $r=0$ ) for larger values of $\lambda$ compared to Case (3.a). This has the following interpretation: If the portfolios of banks are independent, then this creates diversification in the system and reduces risk. Therefore, even a relatively risk-averse central bank (large $\lambda$ ) can pursue aggressive expansionary monetary policy.

(3.c) Correlated portfolio process with same growth rates $\mu=\mu_{i}$ and volatilities $\sigma^{2}=\sigma_{i}^{2}$. Assume the driving Brownian motions of these portfolio process are correlated as in Equation (10). After calculation, we get:

$$
\begin{gathered}
g(r)=\left\{\begin{array}{l}
\frac{(\mu-r)^{2}}{\sigma^{2}}+r, r \leq \mu-\sigma^{2} \\
\mu-\frac{\sigma^{2}}{2}, r \geq \mu-\sigma^{2}
\end{array}\right. \\
\rho^{2}(t):=c\left(\frac{\mu-r}{\sigma} \wedge \sigma\right)^{2}, c:=\lambda\left(\frac{N-1}{N} \rho_{0}+\frac{1}{N}\right) .
\end{gathered}
$$


Then, we can find optimal $r$ : this is

$$
r^{*}=\left\{\begin{array}{l}
0, c<1-2\left(\frac{\mu}{\sigma^{2}}-1\right)^{-1} \\
\mu-\sigma^{2}, c>1-2\left(\frac{\mu}{\sigma^{2}}-1\right)^{-1}
\end{array}\right.
$$

Note that for $\rho_{0}=1$ we get Case (3.a), and for $\rho_{0}=0$ we get Case (3.b). Case (3.c) is intermediate: there is diversification between portfolios of private banks, but this diversification is not complete. Therefore, a risk-averse central bank can pursue more expansionary monetary policy than in Case (3.a), but less so than in Case (3.b).

To illustrate the impact of risk aversion $\lambda$ on the optimal interest rate, we simulate three scenarios. First, in Figure 9, we assume uncorrelated portfolio process, each with same mean and volatility $\mu_{i}=\sigma_{i}=0.1$ for $i=1, \ldots, N$. This is Case (3.a), which is discussed above in this section.

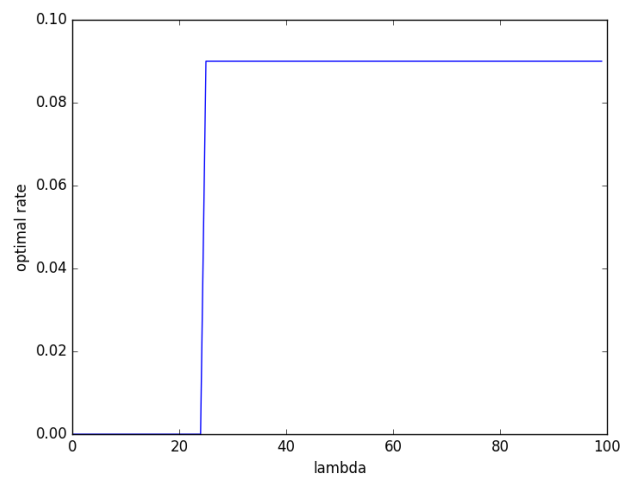

(a) optimal interest rate

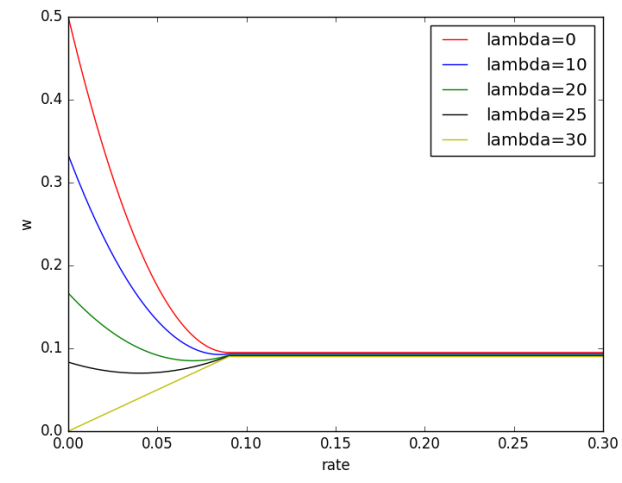

(b) $w(r, \lambda)$

Figure 9. Optimal interest rate with $N=30$ uncorrelated portfolio process: $\rho_{0}=0$, with $\mu_{i}=\sigma_{i}=0.1$ for $i=1, \ldots, N$.

Next, in Figure 10, we assume independent portfolio process but with mean and standard deviation $\mu_{i}, \sigma_{i}, i=1, \ldots, N$ i.i.d uniform on [0.1,0.2]. This is Case (3.b), which is discussed above in this section. However, to our surprise, we observe the optimal interest rate to have only one jump as we increase the risk aversion parameter $\lambda$.

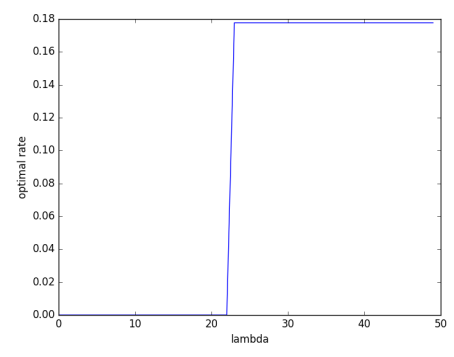

(a) optimal interest rate

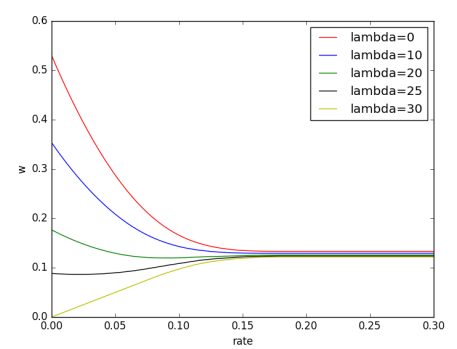

(b) $w(r, \lambda)$

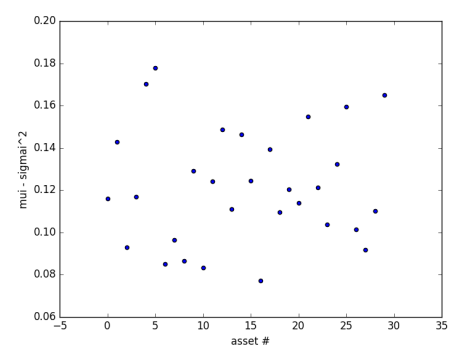

(c) $\mu_{i}-\sigma_{i}^{2}$

Figure 10. Optimal interest rate with $N=30$ uncorrelated assets. with mean and standard deviation $\mu_{i}, \sigma_{i}, i=1, \ldots, N$ i.i.d uniform on $[0.1,0.2]$.

Finally, in Figure 11, we assume correlated portfolio process with $\rho_{0}=0.8$ and mean and standard deviation $\mu_{i}, \sigma_{i}, i=1, \ldots, N$ drawn from i.i.d uniform $[0.1,0.2]$. This is a generalized version of Case (3.c) discussed above. We observe that, due to correlation in the portfolio process, even a relatively less risk averse central bank is forced to raise the interest rate. 


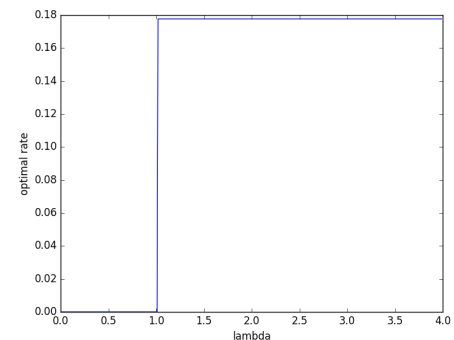

(a) optimal interest rate

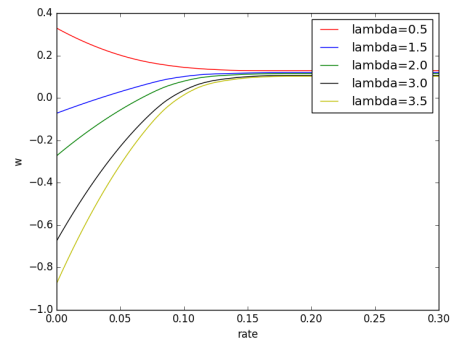

(b) $w(r, \lambda)$

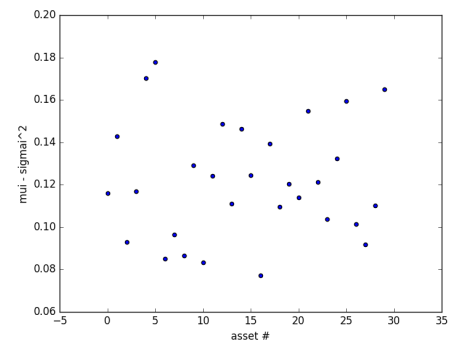

(c) $\mu_{i}-\sigma_{i}^{2}$

Figure 11. Optimal interest rate with $N=30$ portfolio process, with correlation $\rho_{0}=0.8$ and $\mu_{i}, \sigma_{i}, i=1, \ldots, N$ i.i.d uniform on $[0.1,0.2]$.

Remark 7. The optimization problems discussed in this section maximized the expected utility of the central bank. This is in contrast to a more common measure of discounted expected utility $\mathcal{U}$ :

$$
\mathbb{E} \int_{0}^{T} e^{-\gamma t} \mathcal{U}_{\gamma}\left(\bar{Y}^{*}(t)\right) \mathrm{d} t
$$

However, if the discounting term $\gamma$ is not dependent on controlled interest rate $r$, the optimal policy remains the same as proposed in Theorem 2.

Remark 8. In the current formulation, the private banks solve their problems given $r$, and the central bank in anticipation of them solves for $r$ incorporating the future decisions of the private banks. In practice, this is similar to central banks announcing the policy rate to which the private players react. Reversing the order of the solution makes the problem mathematically uninteresting and its economics impractical. Within this scenario, Equations (12) and (13) imply that the optimal interest rate for the central bank $r=0$, thus the central bank is just an observer with no ability to direct the economy. This is in sharp contrast to the real economy where central banks play an active role through the monetary policy.

\section{Long-Term Stability}

In this section, we analyze the long-term behavior of the centered process:

$$
\tilde{Y}=\left(\tilde{Y}_{1}, \ldots, \tilde{Y}_{N}\right), \tilde{Y}_{i}(t)=Y_{i}(t)-\bar{Y}(t), i=1, \ldots, N
$$

It takes values in the hyperplane $\Pi:=\left\{y \in \mathbb{R}^{N} \mid y_{1}+\ldots+y_{N}=0\right\}$. In other words, we are trying to find whether log capitals of banks stay together as time $t$ goes to infinity, or they split into two or more "clouds". The key parameters are rates $c_{i j}$ of interbank cash flows. Under certain fairly general conditions on these rates, the process (49) is ergodic: It has a unique stationary distribution; and for any initial conditions, it converges to this distribution as $t \rightarrow \infty$. This section has two results. Theorem 3 deals with the case of flow rates $c_{i j}$ being time-independent: $c_{i j}(t) \equiv c_{i j}$. Lemma 1 covers the general case.

Assume the central bank has already chosen the interest rate $r=r^{*}$, as above. Then, $\bar{Y}$ is a Brownian motion with drift coefficient $g\left(r^{*}\right)$ and diffusion coefficient $\rho^{2}\left(r^{*}\right)$. We have:

$$
\mathrm{d} Y_{i}(t)=\mathrm{d} M_{i}(t)+\frac{1}{N} \sum_{j=1}^{N} c_{i j}(t)\left(Y_{j}(t)-Y_{i}(t)\right) \mathrm{d} t, i=1, \ldots, N
$$


Here, the process: $M=\left(M_{1}, \ldots, M_{N}\right)$ is an $N$-dimensional Brownian motion with drift vector and covariance matrix $\mu^{*}=\left(\mu_{1}^{*}, \ldots, \mu_{N}^{*}\right)$ and $A^{*}=\left(a_{i j}^{*}\right)_{i, j=1, \ldots, N}$ from Equations (32) and (33). The centered process in Equation (49) satisfies the SDE

$$
\mathrm{d} \tilde{Y}_{i}(t)=\mathrm{d} \tilde{M}_{i}(t)+\frac{1}{N} \sum_{j=1}^{N} c_{i j}(t)\left(\tilde{Y}_{j}(t)-\tilde{Y}_{i}(t)\right) \mathrm{d} t, i=1, \ldots, N .
$$

Here, $\tilde{M}_{i}(t):=M_{i}(t)-\bar{M}(t)$ for $i=1, \ldots, N$. Note that $\tilde{M}=\left(\tilde{M}_{1}, \ldots, \tilde{M}_{N}\right)$ is a $\Pi$-valued Brownian motion. It has drift vector

$$
\tilde{\mu}^{*}=\left(\tilde{\mu}_{1}^{*}, \ldots, \tilde{\mu}_{N}^{*}\right)^{\prime}, \tilde{\mu}_{i}^{*}:=\mu_{i}^{*}-\bar{\mu}^{*}, \bar{\mu}^{*}:=\frac{1}{N} \sum_{i=1}^{N} \mu_{i}^{*}
$$

and covariance matrix

$$
\tilde{A}^{*}=\left(\tilde{a}_{i j}^{*}\right):=V A^{*} V, V=I_{N}-N^{-1} e e^{\prime}, e=(1, \ldots, 1)^{\prime} \in \mathbb{R}^{N} .
$$

Therefore, $\tilde{Y}$ is a Markov process. Denote by $P^{t}(x, \cdot)$ its transition function. Define the following measure norm on $\Pi$ for a function $V: \Pi \rightarrow[1, \infty)$ :

$$
\|v\|_{V}:=\sup _{|f| \leq V}\left|\int_{\Pi} f \mathrm{~d} v\right| .
$$

We denote the Euclidean norm of a vector $x=\left(x_{1}, \ldots, x_{d}\right)^{\prime} \in \mathbb{R}^{d}$ by

$$
\|x\|:=\left[x_{1}^{2}+\ldots+x_{d}^{2}\right]^{1 / 2} .
$$

Theorem 3. Assume the flow rates $c_{i j}(t)=c_{i j}$ are constant. Define the graph $G$ on the set of vertices $\{1, \ldots, N\}: i \leftrightarrow j$ iff $c_{i j}>0$. If $G$ is connected, then:

(a) $\tilde{Y}$ has a unique stationary distribution $\pi$ on $\Pi$, which is multivariate normal.

(b) The transition function satisfies for some constants $c, \lambda, k>0$ :

$$
\left\|P^{t}(x, \cdot)-\pi(\cdot)\right\|_{V} \leq c V(x) e^{-k t}, \quad V(x):=\exp \left(\frac{\lambda}{2}\|x\|^{2}\right) .
$$

(c) For any bounded measurable function $f: \Pi \rightarrow \mathbb{R}$ we have, almost surely:

$$
\lim _{T \rightarrow \infty} \frac{1}{T} \int_{0}^{T} f(\tilde{Y}(s)) \mathrm{d} s=\int_{\Pi} f(y) \pi(\mathrm{d} y) .
$$

Proof. From the properties of solutions of SDE and nondegeneracy of the covariance matrix $\tilde{A}^{*}$ of $M$, we have the following positivity property:

$$
P^{t}(x, C)>0 \text { for all } t>0, x \in \Pi, C \subseteq \Pi \text { with } \operatorname{mes}_{\Pi}(C)>0 .
$$

The generator of $\tilde{Y}$ for all twice continuously differentiable functions $f: \Pi \rightarrow \mathbb{R}$ is given by:

$$
\mathcal{L} f(x):=\left[\tilde{\mu}^{*}+\frac{1}{N} \mathcal{M} x\right] \cdot \nabla f+\frac{1}{2} \sum_{i=1}^{N} \sum_{j=1}^{N} \tilde{a}_{i j}^{*} \frac{\partial^{2} f}{\partial x_{i} \partial x_{j}} .
$$


Here, $\mathcal{M}=\left(m_{i j}\right)_{i, j=1, \ldots, N}$ is the following matrix:

$$
m_{i j}=\left\{\begin{array}{l}
c_{i j}, i \neq j ; \\
-\sum_{k \neq i} c_{i k}, i=j .
\end{array}\right.
$$

Now, plug this function $V$ from Equation (54) for a suitable $\lambda$ into the generator in Equation (56). Then,

$$
\nabla V=\lambda x V, \frac{\partial^{2} V}{\partial x_{i} \partial x_{j}}=\left(\lambda^{2} x_{i} x_{j}+\lambda \delta_{i j}\right) V .
$$

Combining Equation (58) with Equation (56), we get:

$$
\mathcal{L} V=\left[\left(\lambda \tilde{\mu}^{*} \cdot x+\frac{\lambda}{N} x^{\prime} \mathcal{M} x\right)+\frac{1}{2}\left(\lambda^{2}\left(x^{\prime} \tilde{A}^{*} x\right)+\lambda \operatorname{tr}\left(\tilde{A}^{*}\right)\right)\right] V .
$$

Using Lemma A4 below, we get:

$$
\frac{1}{N}\left[x^{\prime} \mathcal{M} x\right] \leq-c_{0}\|x\|^{2}, \quad c_{0}:=\frac{c(\mathcal{M})}{N} .
$$

There exists a constant $a_{0}>0$ such that for all $x \in \Pi$, we have: $x^{\prime} \tilde{A}^{*} x \leq a_{0}\|x\|^{2}$. Combining this observation with Equations (59) and (60), we get:

$$
\mathcal{L} V \leq\left[\lambda \tilde{\mu}^{*} \cdot x-\lambda c_{0}\|x\|^{2}+\frac{1}{2} a_{0} \lambda^{2}\|x\|^{2}+\frac{1}{2} \lambda \operatorname{tr}\left(\tilde{A}^{*}\right)\right] V .
$$

Choose $\lambda:=c_{0} / a_{0}$, then Equation (61) takes the form

$$
\mathcal{L} V(x) \leq K(x) V(x), \quad K(x):=\frac{c_{0}}{a_{0}} \tilde{\mu}^{*} \cdot x-\frac{c_{0}^{2}}{2 a_{0}^{2}}\|x\|^{2}+\frac{1}{2} \frac{c_{0}}{a_{0}} \operatorname{tr}\left(\tilde{A}^{*}\right) .
$$

Note that, as $\|x\| \rightarrow \infty$, we have: $K(x) \rightarrow-\infty$. Therefore, for some constants $c_{1}, c_{2}>0$,

$$
K(x) \leq-c_{1},\|x\| \geq c_{2} .
$$

Recall the definition of the ball $\mathcal{B}\left(c_{2}\right)$ in Equation (5). Since $\mathcal{L} V$ and $V$ are continuous, we have:

$$
\max _{x \in \mathcal{B}\left(c_{2}\right)}\left[\mathcal{L} V(x)+c_{1} V(x)\right]=: c_{3}<\infty .
$$

Combining Equation (62) with Equations (63) and (64), we get:

$$
\mathcal{L} V(x) \leq-c_{1} V(x)+c_{3} 1_{\mathcal{B}\left(c_{2}\right)}(x) .
$$

Finally, combine Equation (65) with the Feller property of $\tilde{Y}$ (i.e., for a bounded continuous function $\mathrm{f}$, the map $x \mapsto P^{t} f(x)$ is also bounded and continuous for the transition function $P$ of $\tilde{Y}$ ), and with the positivity property in Equation (55). Apply Lemma A1 from Appendix A to Lebesgue reference measure $\psi$ and the function $V$ from Equation (54). This completes the proof of (a) (the uniqueness of a stationary distribution), as well as of (b). The fact that this stationary distribution $\pi$ is multivariate normal follows from the observation that $\tilde{Y}$ is a multidimensional Ornstein-Uhlenbeck process on the hyperplane $\Pi$. 
To finish the proof of Theorem 3, let us show (c): This is similar to the proof of (Ichiba et al. 2011, Theorem 1). Take any $r \geq c_{2}$. Adjusting the proof of Equation (65) above, we find that there exists a positive constant $d(r)$ such that

$$
\mathcal{L} V(x) \leq-c_{1} V(x)+d(r) 1_{\mathcal{B}(r)}(x) .
$$

Let $\tau_{\mathcal{B}(r)}:=\inf \{t \geq 0 \mid \tilde{Y}(t) \in \mathcal{B}(r)\}$ be the hitting moment of the ball $\mathcal{B}(r)$, for a fixed $r>0$. Apply (Meyn and Tweedie 1993b, Theorem 4.3(a)), with the function $V$ from Equation (54), with $f:=1$, $\delta:=0$. Then,

$$
\mathbb{E}_{x} \tau_{\mathcal{B}(r)} \leq c_{1}^{-1} V(x), x \in \Pi .
$$

Use the fact that $V$ is bounded on compact subsets to verify assumption (b) in Lemma A2. Assumption (a) of this lemma follows from the observation that the covariance matrix of $\tilde{Y}$ is constant. Now, apply Lemma A2 from (Khasminskii 2012, Theorem 4.1, Theorem 4.2), cited as (Ichiba et al. 2011, Proposition 1). This completes the proof of part (c) of Theorem 3.

Lemma 1. Assume the flow rates are given by

$$
c_{i j}(t)=c_{i j} f(\tilde{Y}(t)), i, j=1, \ldots, N, i \neq j ; t \geq 0,
$$

where $f: \Pi \rightarrow \mathbb{R}$ is a function such that

$$
\varliminf_{\|z\| \rightarrow \infty} f(z)>0,
$$

and $c_{i j}$ are real numbers as in Theorem 3 . Then, the conclusion of Theorem 3 is the same, minus the conclusion that $\pi$ is multivariate normal.

Proof. Similar to Theorem 3, but with the following changes: Instead of Equation (59), we have:

$$
\mathcal{L} V(x)=\left[\lambda \tilde{\mu}^{*} \cdot x+\frac{\lambda f(x)}{N} x^{\prime} \mathcal{M} x+\frac{1}{2}\left(\lambda^{2}\left(x^{\prime} \tilde{A}^{*} x\right)+\lambda \operatorname{tr}\left(\tilde{A}^{*}\right)\right)\right] V .
$$

There exist $c_{4}, c_{5}>0$ such that $f(x) \geq c_{4}$ for $x \in \Pi,\|x\| \geq c_{5}$. Therefore, for such $x$, the estimate (62) is preserved with $c_{0}$ changed to $c_{0} c_{4}$. The rest of the proof is similar to that of Theorem 3.

Note, however, that, if the graph $G$ is disconnected, then this stability breaks down. Indeed, assume $G$ has connected components $G_{1}$ and $G_{2}$ (only two for sake of notational simplicity; analysis is the same for more than two connected components), and the flow rates $c_{i j}$ are positive constants if $i$ and $j$ are adjacent, $c_{i j}=0$ if not. By Theorem 3, we get:

$$
\left(Y_{i}-\bar{Y}_{1}\right)_{i \in G_{1}},\left(Y_{i}-\bar{Y}_{2}\right)_{i \in G_{2}}
$$

are ergodic, that is, they satisfy an inequality similar to Equation (54). Here,

$$
\bar{Y}_{1}(t):=\frac{1}{\left|G_{1}\right|} \sum_{i \in G_{1}} Y_{i}(t), \bar{Y}_{2}(t):=\frac{1}{\left|G_{2}\right|} \sum_{i \in G_{2}} Y_{i}(t)
$$

However, these averages from Equation (66) are, in fact, Brownian motions with certain drift and diffusion coefficients, which are easy to calculate from Equation (50). They are correlated, but not perfectly. Therefore, $\bar{Y}_{1}-\bar{Y}_{2}$ is not ergodic, and the process $\tilde{Y}$ defined in Equation (49) is also not ergodic. Private banks are separated into two groups, which "drift" from each other. 


\section{Concluding Remarks}

We studied a model of $N$ private banks exchanging money through interbank flows, borrowing from the non-banking financial sector under an interest rate set by the central bank, and investing in portfolios consisting of risky assets; these portfolios are modeled by correlated geometric Brownian motions. This represents an enhancement of the model in Equation (1), which is obtained in (Carmona et al. 2013) as a result of banks borrowing from each other. We generalize the interbank flows from (Carmona et al. 2013), making them heterogeneous.

Each private bank maximizes its expected terminal logarithmic utility. The central bank maximizes exponential utility function of the total size of the system. We are able to solve the control problems for each private banks and the central bank because of this special choice of utility functions. The resulting dynamics looks a bit like Equation (1), except that each private bank has its own growth rate and volatility in the driving Brownian motion, and the flow rates $c_{i j}$ depend on $i$ and $j$.

Our setup allowed us to study systemic risk and distribution of defaults under different market and investment scenarios. We also observe common economic phenomena of liquidity trap (where the monetary policy fails to boost the investment in risky assets) naturally arising from the model.

For future research, one can consider the case when some but not all portfolios $S_{i}$ satisfy $\mu_{i} \leq \sigma_{i}^{2}$ (and are therefore unprofitable). In addition, it might be interesting to consider different utility functions for private banks, for example power utility. Since the corresponding Hamilton-Jacobi-Bellman equations are likely to be intractable, the problem might be analyzed using mean-field formulation, each bank is competing against the "mass of banks".

Author Contributions: The authors contributed equally to this work.

Funding: The authors are supported in part by NSF grants DMS 1736439 and DMS 1409434.

Acknowledgments: We are grateful to Rene Carmona, Zachary Feinstein, Jean-Pierre Fouque, Matheus Grasselli, Ioannis Karatzas, Mike Ludkovski, Andreea Minca, and Soumik Pal for useful discussion.

Conflicts of Interest: The authors declare no conflict of interest.

\section{Abbreviations}

The following abbreviations are used in this manuscript:

CARA Constant Relative Risk Aversion

NSF National Science Foundation

SDE Stochastic Differential Equation

\section{Appendix A}

Let us state explicitly convergence results for general continuous-time Markov processes, used in the proof of Theorem 3. These results from classic papers (Down et al. (1995); Meyn and Tweedie 1993a, 1993b) link Lyapunov functions with long-term convergence. In (Sarantsev 2016, Lemma 2.3, Theorem 2.6), we reformulate these results to make them more convenient for our use. Let us restate these results here, for convenience of the reader.

Lemma A1. Take a Feller continuous strong Markov process $\mathfrak{X}=(\mathfrak{X}(t), t \geq 0)$ on the metric state space $\mathcal{E}$, with transition function $P^{t}(x, \cdot)$, and generator $\mathcal{L}$. Denote by $\mathbf{P}_{x}$ the probability measure under which $\mathfrak{X}(0)=x$. Assume for some positive reference measure $\psi$ and a function $V: \mathcal{E} \rightarrow[1, \infty)$ in the domain $\mathcal{D}(\mathcal{L})$ of the generator $\mathcal{L}$, we have:

(a) For some compact subset $C \subseteq \mathcal{E}$, we have $\psi(C)>0$.

(b) For all $\psi$-positive subsets $A \subseteq \mathcal{E}, x \in \mathcal{E}, t>0$, we have: $P^{t}(x, A)>0$.

(c) For some constants $b, k>0$ and a compact set $K \subseteq \mathcal{E}$, we have:

$$
\mathcal{L} V(x) \leq-k V(x)+b 1_{K}(x), x \in \mathcal{E} ; \text { and } \sup _{x \in K} V(x)<\infty .
$$


Then, there exists a unique stationary distribution $\pi$, and the transition function satisfies the following estimate: for some constants $D, \varkappa>0$,

$$
\left\|P^{t}(x, \cdot)-\pi(\cdot)\right\|_{V} \leq D V(x) e^{-\varkappa t}, x \in \mathcal{E}, t \geq 0 .
$$

The following Strong Law of Large Numbers is taken from (Khasminskii 2012, Theorem 4.1, Theorem 4.2). It holds under an assumption which can be called uniform positive recurrence, and which can be deduced from existence of Lyapunov functions. Assume that $\mathcal{E}=\mathbb{R}^{d}$ above, and $\mathcal{X}$ is the solution of an SDE with a certain drift vector, and the covariance matrix $\mathcal{A}(\cdot)$. Let $\tau_{C}:=\inf \{t \geq 0 \mid \mathfrak{X}(t) \in C\}$ be the hitting time of a subset $C \subseteq \mathbb{R}^{d}$. Assume there exists a unique stationary distribution $\pi$.

Lemma A2. Assume for some open bounded domain $D \subseteq \mathcal{E}$ with $C^{2}$ boundary, we have:

(a) The smallest eigenvalue of $\mathcal{A}(x)$ for $x \in \bar{D}$ is uniformly bounded away from zero.

(b) For every compact subset $\mathcal{K} \subseteq \mathbb{R}^{d}$, we have: $\sup \mathbb{E}_{x} \tau_{D}<\infty$.

Then, $\mathbf{P}_{x}$-a.s. for every $x \in \mathbb{R}^{d}$ and bounded measurable function $f: \mathbb{R}^{d} \rightarrow \mathbb{R}$, we have:

$$
\lim _{T \rightarrow \infty} \frac{1}{T} \int_{0}^{T} f(\mathfrak{X}(t)) \mathrm{d} t=\int_{\mathbb{R}^{d}} f(x) \pi(\mathrm{d} x) .
$$

Lemma A3. Fix $\mu \in \mathbb{R}$ and $\sigma>0$. Take a function $h: \mathbb{R} \rightarrow \mathbb{R}$, defined as

$$
h(x)=\mu x-\frac{\sigma^{2}}{2} x^{2}-r(x-1)_{+} .
$$

Its global maximum is reached at the point $x^{*}$ and is equal to $h^{*}=h\left(x^{*}\right)$, where:

$$
h\left(x^{*}\right):=\left\{\begin{array}{l}
r+\frac{(\mu-r)^{2}}{2 \sigma^{2}}, \mu-\sigma^{2} \geq r ; \\
\mu-\frac{\sigma^{2}}{2}, 0 \leq \mu-\sigma^{2} \leq r ; \\
\frac{\mu}{2 \sigma^{2}}, \mu \leq \sigma^{2} .
\end{array} \quad x^{*}:=\left\{\begin{array}{l}
\frac{\mu-r}{\sigma^{2}}, \mu-\sigma^{2} \geq r ; \\
1,0 \leq \mu-\sigma^{2} \leq r \\
\frac{\mu}{\sigma^{2}}, \mu \leq \sigma^{2} .
\end{array}\right.\right.
$$

Proof. We can write

$$
h(x)=\left\{\begin{array}{l}
\mu x-\frac{\sigma^{2}}{2} x^{2}-r(x-1), x \geq 1 ; \\
\mu x-\frac{\sigma^{2}}{2} x^{2}, x \leq 1 .
\end{array}\right.
$$

First, note that the function $h$ is smooth everywhere except $x=1$, and $h^{\prime \prime}(x)<0$ for all $x \neq 1$. Therefore, if $h^{\prime}(x)=0$, then $h$ has a local maximum at $x$. Take derivatives on both intervals $(-\infty, 1]$ and $[1, \infty)$ :

$$
\begin{aligned}
& x \geq 1 \quad \text { implies } \quad h^{\prime}(x)=(\mu-r)-\sigma^{2} x=0 \Longrightarrow x=x_{1}:=\frac{\mu-r}{\sigma^{2}} \\
& x \leq 1 \quad \text { implies } \quad h^{\prime}(x)=\mu-\sigma^{2} x=0 \Longrightarrow x=x_{2}:=\frac{\mu}{\sigma^{2}} .
\end{aligned}
$$

On both these rays, $h$ is a parabola with branches facing down.

Case 1. $\mu-\sigma^{2} \geq r$. Then, $x_{1}, x_{2} \geq 1$. Therefore, $h$ reaches maximum on $[1, \infty)$ at $x_{1}$, and on $(-\infty, 1]$ at 1. Since $h$ reaches its maximum on $[1, \infty)$ at $x_{1}$ and not 1 , we have: $h(1) \leq h\left(x_{1}\right)$. As a result, $x^{*}=x_{1}$. Case 2. $0 \leq \mu-\sigma^{2} \leq r$. Then, $x_{1} \leq 1$, but $x_{2} \geq 1$. Therefore, $h$ reaches maximum on $[1, \infty)$ at 1 , and on $(-\infty, 1]$ at 1 . As a result, the global maximum will be at $x^{*}=1$. 
Case 3. $\mu-\sigma^{2} \leq 0$. Then, $x_{1}, x_{2} \leq 1$. Therefore, $h$ reaches maximum on $[1, \infty)$ at 1 , and on $(-\infty, 1]$ at $x=x_{2}$. Similar to Case 1 , the global maximum is reached at $x^{*}=x_{2}$.

Lemma A4. For the matrix $\mathcal{M}$ defined in (57), there exists a constant $c(\mathcal{M})>0$ such that

$$
x^{\prime} \mathcal{M} x \leq-c(\mathcal{M})\|x\|^{2}, \quad x \in \Pi .
$$

Proof. Note that $\mathcal{M}$ is a generator matrix for a continuous-time Markov chain $Q=(Q(t), t \geq 0)$ on $\{1, \ldots, N\}$. This Markov chain can be viewed as a biased random walk on the graph $G$ : As it wants to jump out of a state $i$, it chooses one of its nearest neighbors $j$, such that $i$ and $j$ are connected, only not with uniform probability. This graph $G$ is connected. Therefore, this Markov chain is irreducible. Since it is finite, it is positive recurrent. From the standard results on continuous-time Markov chains, see for example (Suhov and Kelbert 2008, Theorem 2.7.15), this Markov chain $Q$ has a unique stationary distribution

$$
\pi^{Q}=\left[\begin{array}{ccc}
\pi_{1}^{Q} & \ldots & \pi_{N}^{Q}
\end{array}\right]
$$

This stationary distribution satisfies $\pi^{Q} \mathcal{M}=0$. However, the columns of the matrix $\mathcal{M}$ sum up to zero. Therefore, $e^{\prime} \mathcal{M}=0$, and $e / N$ is a stationary distribution. By uniqueness, $\pi^{Q}=e / N$. Let $\lambda_{1}, \ldots \lambda_{N}$ and $v_{1}, \ldots, v_{N}$ be the eigenvalues and eigenvectors of the matrix $\mathcal{M}$

$$
\mathcal{M} v_{i}=\lambda_{i} v_{i}, i=1, \ldots, N \text {. }
$$

The eigenvectors of the matrix $\mathcal{M}$ are all real, because $\mathcal{M}$ is symmetric. Next, nonzero eigenvalues are negative: This follows from (Iosifescu 2007, Exercise 8.1). Each zero eigenvalue $\lambda_{i}$ has eigenvector $v_{i}$ which satisfies $v_{i}^{\prime} \mathcal{M}=0$, that is, $v_{i}^{\prime}$ is proportional to a stationary distribution. However, the stationary distribution is unique, so we have (without loss of generality):

$$
\lambda_{1}=0 ; \lambda_{2}, \ldots, \lambda_{N}<0 ; v_{1}=c e \text { for some constant } c .
$$

Now, take an $x \in \mathbb{R}^{N}$. Assume $v_{1}, \ldots, v_{N}$ are normalized: $\left\|v_{i}\right\|=1, i=1, \ldots, N$. Because $\mathcal{M}$ is symmetric, $v_{1}, \ldots, v_{N}$ form an orthonormal basis in $\mathbb{R}^{N}$. Therefore, we can decompose

$$
x=\left(x \cdot v_{1}\right) v_{1}+\left(x \cdot v_{2}\right) v_{2}+\ldots+\left(x \cdot v_{N}\right) v_{N} .
$$

For a vector $x \in \Pi$, we have: $x \cdot e=0$, and therefore $x \cdot v_{1}=0$. Thus, Equation (A3) takes the form

$$
x=\left(x \cdot v_{2}\right) v_{2}+\ldots+\left(x \cdot v_{N}\right) v_{N} .
$$

Apply the matrix $M$ to this vector in Equation (A4) and use Equation (A2). We have:

$$
\mathcal{M} x=\left(x \cdot v_{2}\right) \lambda_{2} v_{2}+\ldots+\left(x \cdot v_{N}\right) \lambda_{N} v_{N} .
$$

From Equations (A4) and (A5), since $v_{1}, \ldots, v_{N}$ form an orthonormal basis of $\mathbb{R}^{N}$, we have:

$$
x^{\prime} \mathcal{M} x=\mathcal{M} x \cdot x=\lambda_{2}\left(x \cdot v_{2}\right)^{2}+\ldots+\lambda_{N}\left(x \cdot v_{N}\right)^{2} .
$$

In addition, multiplying Equation (A4) by itself, we get:

$$
\|x\|^{2}=x \cdot x=\left(x \cdot v_{2}\right)^{2}+\ldots+\left(x \cdot v_{N}\right)^{2} .
$$

Let $c(\mathcal{M}):=\min \left(\left|\lambda_{2}\right|, \ldots,\left|\lambda_{N}\right|\right)>0$. Comparing Equations (A6) and (A7), we get Equation (A1). 


\section{References}

Banerjee, Tathagata, Alex Bernstein, and Zachary Feinstein. 2018. Dynamic clearing and contagion in financial networks. arXiv. arXiv:1801.02091.

Carmona, René. 2016. Lectures on BSDEs, Stochastic Control, and Stochastic Differential Games with Financial Applications. Philadelphia: SIAM Press.

Carmona, René, François Delarue, and Daniel Lacker. 2016. Mean field games with common noise. The Annals of Probability 44: 3740-803. [CrossRef]

Carmona, René, Jean-Pierre Fouque, Seyyed Mostafa Mousavi, and Li-Hsien Sun. 2018. Systemic risk and stochastic games with delay. Journal of Optimization Theory and Applications 179: 366-99. [CrossRef]

Carmona, Rene, Jean-Pierre Fouque, and Li-Hsien Sun. 2013. Mean-Field Games and Systemic Risk. arXiv. arXiv:1308.2172.

Carmona, René, and Daniel Lacker. 2015. A probabilistic weak formulation of mean field games and applications. The Annals of Applied Probability 25: 1189-231. [CrossRef]

Down, Douglas, Sean P. Meyn, and Richard L. Tweedie. 1995. Exponential and uniform ergodicity for markov processes. The Annals of Probability 23: 1671-91. [CrossRef]

Eisenberg, Larry, and Thomas H. Noe. 2001. Systemic risk in financial systems. Management Science 47: $236-49$. [CrossRef]

Feldman, David. 1992. Logarithmic preferences, myopic decisions, and incomplete information. Journal of Financial and Quantitative Analysis 27: 619-29. [CrossRef]

Fouque, Jean-Pierre, and Joseph A. Langsam, eds. 2013. Handbook on Systemic Risk. Cambridge: Cambridge University Press.

Ichiba, Tomoyuki, Vassilios Papathanakos, Adrian Banner, Ioannis Karatzas, and Robert Fernholz. 2011. Hybrid atlas models. The Annals of Applied Probability 21: 609-44. [CrossRef]

Iosifescu, Marius. 2007. Finite Markov Processes and Their Applications. Mineola: Dover Publications.

Khasminskii, Rafail. 2012. Stochastic Stability of Differential Equations. Stochastic Modeling and Applied Probability. Berlin: Springer.

Kley, Oliver, Claudia Klüppelberg, and Lukas Reichel. 2015. Systemic risk through contagion in a core-periphery structured banking network. In Advances in Mathematics of Finance. Stettner Będlewo: Banach Center Publications.

Lacker, Daniel. 2016. A general characterization of the mean field limit for stochastic differential games. Probability Theory and Related Fields 165: 581-648. [CrossRef]

Machina, Mark, and W. Kip Viscusi, eds. 2014. Handbook of the Economics of Risk and Uncertainty. Amsterdam: North-Holland, vol. 1.

Meyn, Sean P., and Richard L. Tweedie. 1993a. Stability of Markovian processes II: Continuous-time processes and sampled chains. Advances in Applied Probability 25: 487-517. [CrossRef]

Meyn, Sean P., and Richard L. Tweedie. 1993b. Stability of Markovian processes III: Foster-Lyapunov criteria for continuous-time processess. Advances in Applied Probability 25: 518-48. [CrossRef]

Sarantsev, Andrey. 2016. Reflected Brownian motion in a convex polyhedral cone: Tail estimates for the stationary distribution. Journal of Theoretical Probability 30: 1200-23. [CrossRef]

Suhov, Yuri, and Mark Kelbert. 2008. Probability and Statistics by Example II. Markov Chains: A Primer in Random Processes and Their Applications. Cambridge: Cambridge University Press.

Sun, Li-Hsien. 2018. Systemic risk and interbank lending. Journal of Optimization Theory and Applications 179: 400-24. [CrossRef]

(C) 2018 by the authors. Licensee MDPI, Basel, Switzerland. This article is an open access article distributed under the terms and conditions of the Creative Commons Attribution (CC BY) license (http:/ / creativecommons.org/licenses/by/4.0/). 